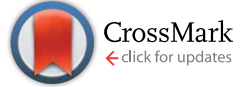

Cite this: RSC Adv., 2016, 6, 108164
Received 13th July 2016

Accepted 16th October 2016

DOI: 10.1039/c6ra17819g

www.rsc.org/advances

\section{An FT-IR spectroscopic study of the role of hydrogen bonding in the formation of liquid crystallinity for mixtures containing bipyridines and 4-pentoxybenzoic acid $\dagger$}

\author{
Alfonso Martinez-Felipe, ${ }^{\text {a }}$ Andrew G. Cook, ${ }^{\mathrm{b}}$ Jordan P. Abberley, ${ }^{\mathrm{b}}$ Rebecca Walker, ${ }^{\mathrm{b}}$ \\ John M. D. Storey ${ }^{b}$ and Corrie T. Imrie ${ }^{b}$
}

\begin{abstract}
We have studied the relationships between liquid crystallinity and hydrogen bonding in mixtures containing 4pentoxybenzoic acid, 5OBA, and five bipyridines, XBiPy, with spacers having different flexibilities, namely: 4,4'bipyridine (BiPy), 1,2-bis(4-pyridyl)ethane (EthaBiPy), trans-1,2-bis(4-pyridyl)ethylene (EthylBiPy), 4,4' trimethylene-dipyridine (PropBiPy), and 4,4'-tetramethylene-dipyridine (ButBiPy). The XBiPy/5OBA mixtures exhibit liquid crystal behaviour for a range of acid concentrations, and predominantly smectic behaviour. The $1: 2$ mixtures have been studied using temperature-dependent Fourier transform infrared spectroscopy, FT$\mathrm{IR}$, with particular focus on the Fermi bands associated with the hydroxyl groups and on the carbonyl stretching regions. The latter has been interpreted in terms of contributions arising from a number of species, namely the heterocomplex, and free, dimeric and oligomeric acid species. This analysis reveals that the heterocomplexes formed by hydrogen bonding between the unlike species tend to be the dominant species but at all temperatures coexist in equilibria with acid monomers, dimers and oligomers. The hydrogen bond strength in the heterocomplexes is predicted by molecular modelling to be stronger than that in the acid dimers. The smectic behaviour of these mixtures is accounted for in terms of the stabilisation of the layered structures arising from hydrogen bonded catemer strands. The liquid crystal behaviour of these and similar hydrogen bonded liquid crystals, HBLCs, cannot be explained solely by the formation of the heterocomplex between the unlike species, but instead a more realistic view must take into account the presence and concentrations of various different supramolecular species and the interactions between them.
\end{abstract}

\section{Introduction}

The overwhelming majority of low molar mass liquid crystals consist of molecules containing a central semi-rigid core, normally comprising phenyl rings connected by unsaturated linkages, attached to which are one or two terminal alkyl chains, see Fig. 1(a). In essence, interactions between the mesogenic cores

${ }^{a}$ Chemical and Materials Engineering Research Group, School of Engineering, University of Aberdeen, King's College, Old Aberdeen AB24 3UE, UK. E-mail: a.martinez-felipe@abdn.ac.uk

${ }^{b}$ Department of Chemistry, School of Natural and Computing Sciences, University of Aberdeen, King's College, Old Aberdeen AB24 3UE, UK

$\dagger$ Electronic supplementary information (ESI) available. See DOI: 10.1039/c6ra17819g promote liquid crystallinity while the role of the chains is to depress the melting point and to give rise to smectic behaviour. Conventionally these molecules are assembled using covalent bonds. Hydrogen bonded liquid crystals, HBLCs, combine the principles of supramolecular chemistry with mesogenic behaviour. The induction or stabilisation of liquid crystallinity in such systems is most often attributed to the enhancement of the structural anisotropy of the resulting complexes with respect to that of the individual components, see Fig. 1. The directionality of the hydrogen bond allows tuning the shapes of the supramolecules while avoiding time consuming and expensive synthetic steps. ${ }^{\mathbf{1 , 2}}$

The first examples of HBLCs, the $n$-alkoxybenzoic acids, were reported by Gray and Jones ${ }^{3-5}$ some 60 years ago. The liquid

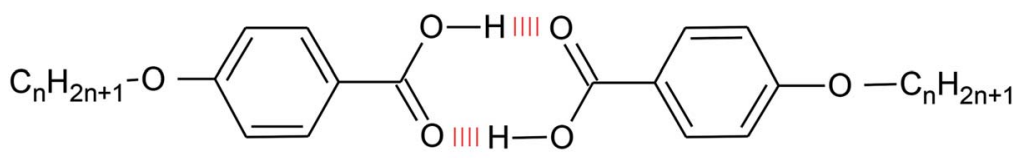


(a)

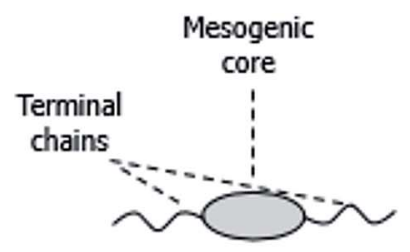

(b)

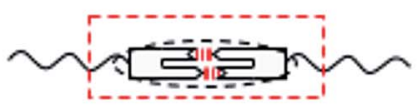

(c)

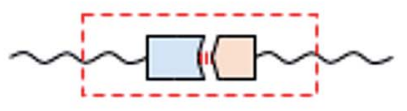

(d)

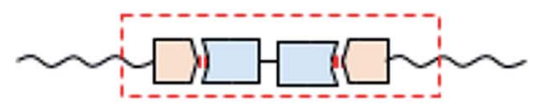

(e)

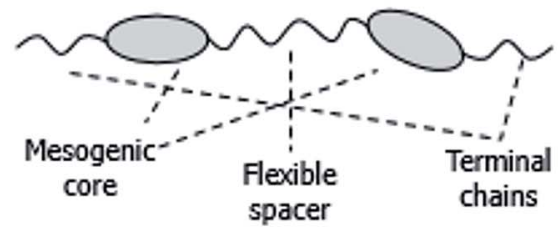

(f)

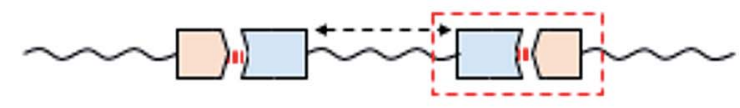

(g)

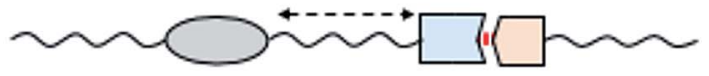

(h)

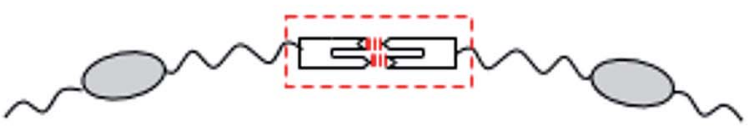

Fig. 1 Schematic representations of covalent and hydrogen bonded liquid crystals: (a) conventional covalent low molar mass; (b) homomeric and (c) heteromeric hydrogen-bonded; (d) bifunctional hydrogen bond donor/acceptor and complimentary monofunctional hydrogen bond acceptor/donor; (e) conventional covalent liquid crystal dimer; (f) symmetric and (g) non-symmetric hydrogen bonded supramolecular dimers; (h) supramolecular trimer.

crystallinity exhibited by these was attributed to the assembly of hydrogen bonded dimers with a supramolecular core formed by the two acid molecules, $\mathbf{1}$, shown schematically in Fig. 1(b), and this homologous series remains the focus of considerable research activity, see for example. ${ }^{6-10}$

This concept in molecular engineering was later extended by Kato and Frechet, who mixed unlike hydrogen bond donors and hydrogen bond acceptors resulting in hydrogen bonded heterocomplexes, 2, and this approach is sketched in Fig. 1(c)..$^{11,12}$

These investigations subsequently triggered the preparation of many different supramolecular liquid crystals via molecular recognition. ${ }^{1,2}$ For example, extended supramolecular cores can be obtained in $2: 1$ complexes formed by linear monofunctional hydrogen bond donors, such as alkoxybenzoic acids, with bifunctional bipyridyl hydrogen bond acceptors, see Fig. 1 (d). ${ }^{13}$

Hydrogen bonding has particular advantages in the preparation of supramolecular dimeric liquid crystals. Conventional covalent liquid crystal dimers consist of molecules containing two mesogenic groups linked via a flexible alkyl spacer, ${ }^{14,15}$ see Fig. 1(e). Liquid crystal dimers can broadly be divided into two classes, namely, symmetric and nonsymmetric. In a symmetric dimer the two mesogenic groups are the same whereas in a non-symmetric dimer they differ. These were initially studied as model compounds for liquid crystal polymers ${ }^{16,17}$ although it soon became apparent that they were of fundamental interest in their own right. Indeed their study resulted in the discovery of intercalated smectic phases ${ }^{18-21}$ and very recently, of the twist-bend nematic phase. ${ }^{22-24}$ Higher liquid crystal oligomers such as trimers containing three mesogenic groups and two spacers ${ }^{25,26}$ and tetramers having four liquid crystal groups and three spacers ${ }^{27,28}$ have also been shown to exhibit fascinating phase behaviour including the twist-bend nematic phase. ${ }^{29-31}$ The first examples of supramolecular symmetric liquid crystal dimers were reported by Kato and co-workers, and consisted of 2:1 complexes assembled using a bifunctional nonmesogenic dicarboxylic acid and extended stilbazole moieties, $3^{32}$ This approach was inverted by Yu and Pan, who reported complexes formed by mixing a central dimeric stilbazole-based hydrogen bond donor with an alkanoic acid 4. ${ }^{33}$

Wallage and Imrie extended this approach to include nonsymmetric dimers by mixing a pyridyl-based hydrogen bond acceptor attached via a flexible spacer to a conventional azobenzene-based mesogenic unit, with an alkoxybenzoic acid, 5 , and this is sketched in Fig. $1(\mathrm{~g})$. $^{34}$

A wide variety of supramolecular liquid crystal dimers have now been studied and their behaviour may be regulated by varying the spacer length and the structures of the individual components. For example, cholesteric phases can be induced by incorporating short spacers containing chiral centres, ${ }^{35}$ or by attaching a cholesterol-based moiety at one end of the complex. ${ }^{36}$ In a different approach in which we control

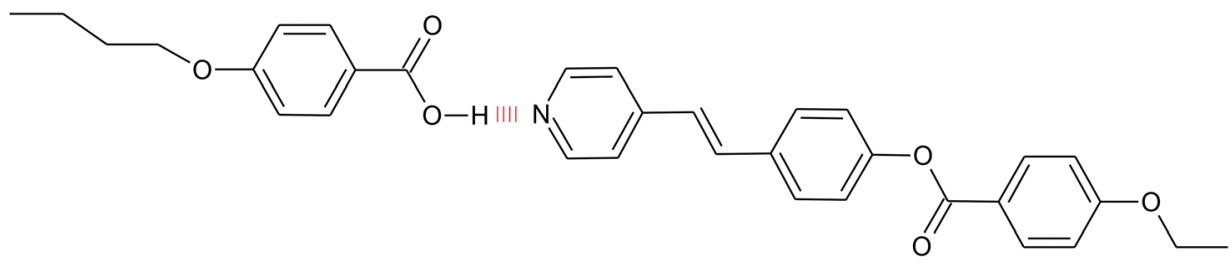



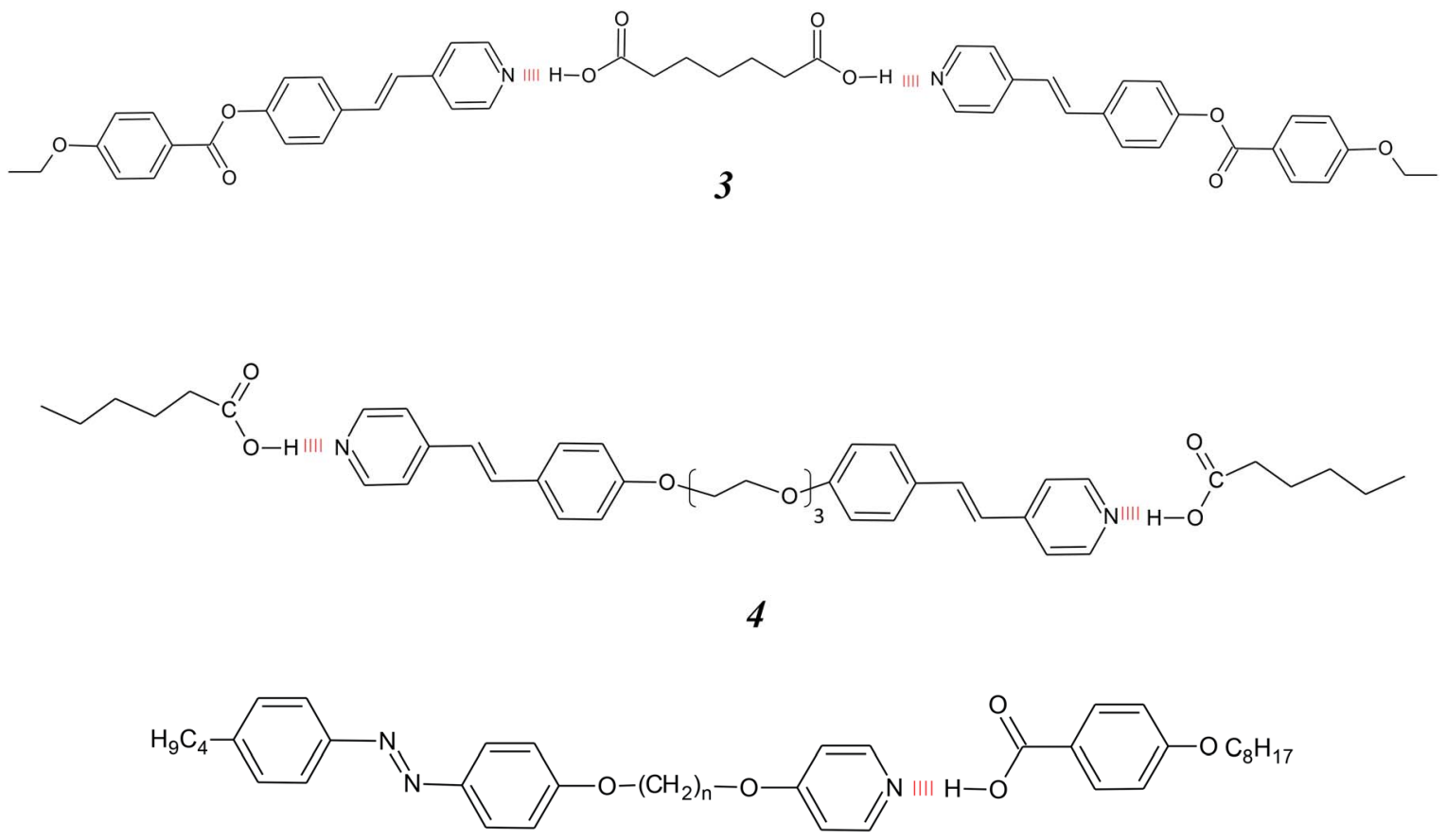

molecular shape using hydrogen bonding, we have recently reported the first example of a supramolecular trimer, 6 and sketched in Fig. 1(h), to show the twist-bend nematic phase. ${ }^{37}$ considerations, but also on the strength of the hydrogen bond..$^{38-40}$ As part of a systematic study of supramolecular liquid crystals, here we report the phase behaviour for mixtures of 4-pentoxybenzoic acid, 5ОВA, as the hydrogen<smiles></smiles>

The liquid crystalline behaviour of HBLCs is most often explained by invoking the quantitative formation of the proposed homo- or heterocomplex, see Fig. 1. It has been shown recently, however, that the formation of supramolecular dimeric (and oligomeric) architectures does not rely only on the composition of the system and geometric

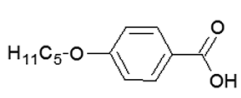

$5 O B A$

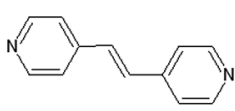

EthylBipy

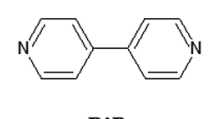

BiPy

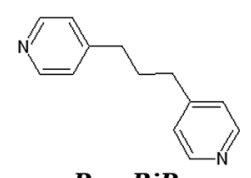

PropBiPy

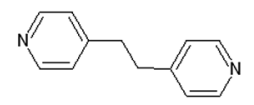

EthaBiPy

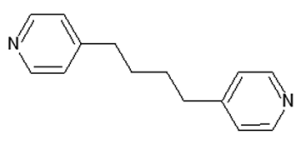

ButBiPy
Fig. 2 Chemical structures and acronyms of the hydrogen bond donor, 5OBA, and the bifunctional hydrogen bond acceptors, XBiPy. bond donor, with five different bifunctional pyridyl-based derivatives, XBiPy, serving as the hydrogen bond acceptors. The structures of these components and the acronyms used to refer to them are shown in Fig. 2. The hydrogen bond acceptors were selected specifically to include both rigid and flexible bipyridyls, and also with the expectation of obtaining linear and bent heterocomplexes. We have also performed a detailed temperature dependent Fourier transform infrared spectroscopic study, FT-IR, of each of the 1:2-XBiPy/5OBA mixtures, and underpinned these studies using molecular modelling. The present study provides further insights into the relationships between hydrogen bonding and mesomorphism, as part of an on-going programme to design novel HBLCs having tailored properties and functionalities.

\section{Experimental section}

\subsection{Materials}

4-Pentoxybenzoic acid (5OBA), 4,4'-bipyridine (BiPy), 1,2bis(4-pyridyl)ethane (EthaBiPy), trans-1,2-bis(4-pyridyl)ethylene 
(EthylBiPy), and 4,4'-trimethylene-dipyridine (PropBiPy) were commercially available and used as received from Sigma Aldrich. The synthesis of 4,4'-tetramethylene-dipyridine (ButBiPy) is described in detail elsewhere. ${ }^{41}$ The mixtures were prepared by co-dissolving appropriate weights of each component in THF and allowing the solvent to evaporate slowly. The complexes were further dried under vacuum for at least 24 hours prior to characterisation. A Mettler Toldedo Microbalance with readability down to $1 \mu \mathrm{g}$ was used to weigh out the samples. The mole fractions listed for each mixture are the calculated values based on actual masses and are rounded to one decimal place.

\subsection{Phase behaviour}

The phase behaviour of the materials was determined by differential scanning calorimetry, DSC, using a Mettler-Toledo DSC820 fitted with an intracooler calibrated with indium and zinc as standards. Heating and cooling rates of $10{ }^{\circ} \mathrm{C} \mathrm{min}-1$ were applied under a nitrogen atmosphere, and all samples were measured in duplicate. Phase assignments were made by polarised light microscopy (POM), using an Olympus BH2 microscope equipped with a Linkam TMHS 600 heating stage and a TMS 91 or 92 control unit.

\subsection{Temperature dependent Fourier transform infrared spectroscopy (FT-IR)}

Temperature dependent infrared spectra were collected using a Nicolet Nexus bench attached to a Continuum FT-IR microscope (Thermo Nicolet) equipped with a Linkam FT-IR 600 heating stage and a TMS 93 control unit. The FT-IR spectra were recorded in trans-reflectance mode, i.e., the IR beam passed through the sample, was reflected by the gold surface back to the sample and then detected (reflectance-absorption). All data were obtained in isothermal steps on cooling, in the 4000-400 $\mathrm{cm}^{-1}$ range, with a resolution of $4 \mathrm{~cm}^{-1}$, and collected after 64 scans.

\subsection{Molecular modelling}

Quantum mechanical density functional theory (DFT) calculations $\mathrm{s}^{42}$ were used to determine the geometric and electronic properties of the hydrogen bonded complexes. Initially, geometric optimisation was performed on the 5OBA acid dimer in both an open and closed conformation using Gaussian G09W at the B3LYP/6-31G(d) level of theory. Using one monomer unit from the optimised closed dimer pair as an initial conformation for the 5OBA segments of the hydrogen bonded benzoic acid:pyridine heterocomplexes, these were also optimised at the B3LYP/6-31G(d) level. In order to estimate the hydrogen bond dissociation energy, the interatomic distances for the hydrogen participating in the hydrogen bond and the hydrogen bond acceptor was set to approximately $5 \AA$ and a single point energy calculation was performed, also at the $\mathrm{B} 3 \mathrm{LYP} / 6-31 \mathrm{G}(\mathrm{d})$ level of theory. At this distance it is reasonable to assume that the hydrogen bond is essentially broken ${ }^{43}$ and so the difference in the energies will provide an estimate of the hydrogen bond strength. For visualisation of space filling models QuteMol was used, ${ }^{44}$ and for visualisation of electrostatic potential isosurfaces, ball-and-stick models and dipole moments, GaussView 5 was used. ${ }^{45}$

\section{Results and discussion}

\subsection{Phase behaviour of components and mixtures}

Table 1 lists the transitional properties of 4-pentoxybenzoic acid, 5OBA, and the bypyridyl-based derivatives, XBiPy, shown in Fig. 2. 5OBA exhibits a nematic phase whereas all the bipyridines under study melt directly from the crystal into the isotropic phase. The phase diagrams of the XBiPy/5OBA mixtures are shown in Fig. 3, and liquid crystallinity was observed for selected concentrations in each set of mixtures. Nematic phases were identified using POM on the basis of the observation of a characteristic Schlieren optical texture containing both types of point singularity, and which flashed when subjected to mechanical stress. For smectic A, SmA, phases, bâtonnets developed on cooling at the isotropicsmectic A or nematic-smectic A transition, and which coalesced to give focal conic fan textures in coexistence with homeotropic regions. For the 1:2-BiPy/5OBA mixture, on cooling the smectic A phase a Schlieren texture developed from regions of homeotropic alignment characteristic of the smectic C phase. The monotropic nature of this phase precluded its characterisation using other techniques. Typical examples of the optical textures observed for these mixtures are shown in Fig. 4.

At the highest acid concentration each XBiPy/5OBA mixture shows exclusively nematic behaviour, see Fig. 3. On decreasing the acid concentration, smectic behaviour emerges in each set of mixtures with the exception of the PropBiPy/5OBA system. For the mixtures containing rigid hydrogen bond acceptors (BiPy, EthylBiPy) smectic behaviour is strongly favoured as the concentration of the hydrogen bond acceptor is increased. Similar behaviour is observed for the more flexible, but essentially semi-rigid, EthaBiPy/5OBA system. Fig. 5 compares the liquid crystal-isotropic transition temperatures for each set of mixtures, and the clearing temperature increases in the order:

$$
\begin{aligned}
\text { PropBiPy } / 5 \mathrm{OBA} & <\mathrm{ButBiPy} / 5 \mathrm{OBA}<\mathrm{EthaBiPy} / 5 \mathrm{OBA} \\
& <\mathrm{BiPy} / 5 \mathrm{OBA}<\text { EthylBiPy } / 5 \mathrm{OBA}
\end{aligned}
$$

Table 1 Transition temperatures and associated entropy changes of the individual components ( $\mathrm{Cr}$, crystal; $\mathrm{N}$, nematic; I, isotropic)

\begin{tabular}{lll}
\hline & $T_{\mathrm{Cr}-\mathrm{I}} /{ }^{\circ} \mathrm{C},{ }^{\mathrm{a}} T_{\mathrm{Cr}-\mathrm{N}} /{ }^{\circ} \mathrm{C},{ }^{\mathrm{b}} T_{\mathrm{N}-\mathrm{I}} /{ }^{\circ} \mathrm{C}$ & $\Delta S_{\mathrm{Cr}-\mathrm{I}} / R,{ }^{\mathrm{a}} \Delta S_{\mathrm{Cr}-\mathrm{N}} / R ;{ }^{\mathrm{b}} \Delta S_{\mathrm{N}-\mathrm{I}} / R$ \\
\hline 5OBA & ${ }^{\mathrm{a}} 125,{ }^{\mathrm{b}} 151$ & $6.46,{ }^{\mathrm{b}} 0.54$ \\
BiPy & 113 & 5.47 \\
EthylBiPy & 153 & 6.62 \\
EthaBiPy & 112 & 7.72 \\
PropBiPy & 60 & 9.71 \\
ButBiPy & 112 & 10.27
\end{tabular}


$\mathrm{T} /{ }^{\circ} \mathrm{C}$

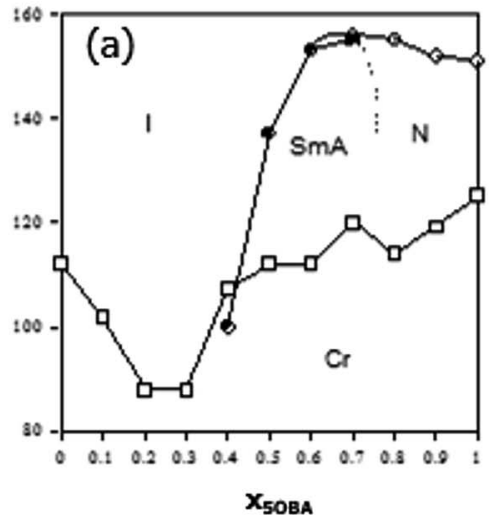

$\mathrm{T} /{ }^{\circ} \mathrm{C}$

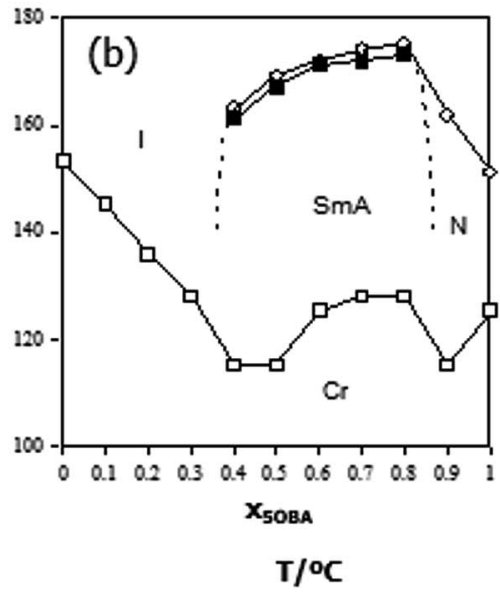

$\mathbf{T} /{ }^{\circ} \mathrm{C}$

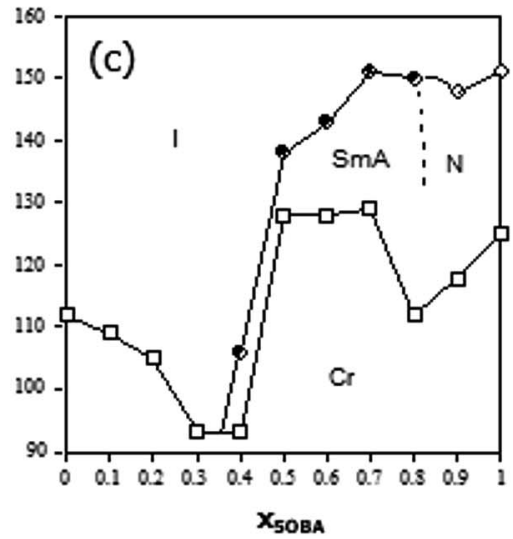

$\mathbf{T} /{ }^{\circ} \mathrm{C}$

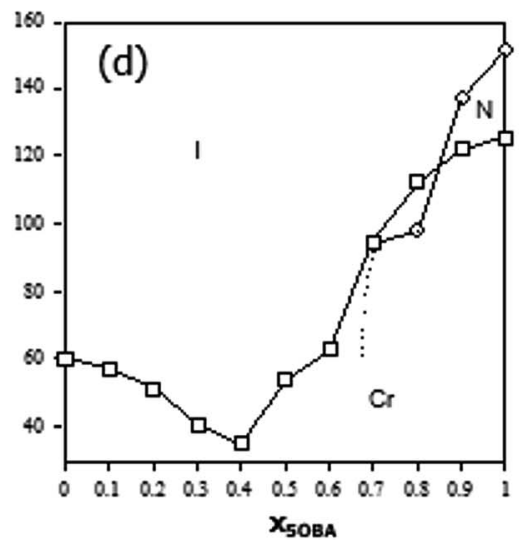

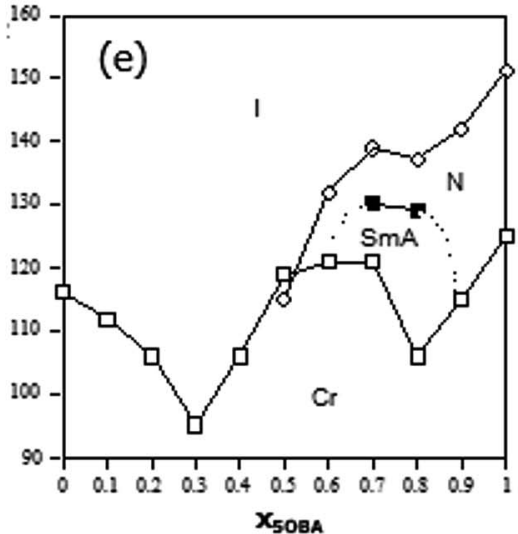

Fig. 3 Phase diagrams of the XBiPy/5OBA mixtures plotted as a function of the mole fraction of 5OBA, $x_{50 B A}$ : (a) BiPy/5OBA; (b) EthylBiPy/5OBA; (c) EthaBiPy/5OBA; (d) PropBiPy/5OBA, and (e) ButBiPy/5OBA (I: isotropic; N: nematic; SmA: smectic A; Cr: crystal).

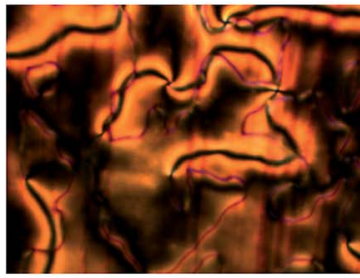

(a)

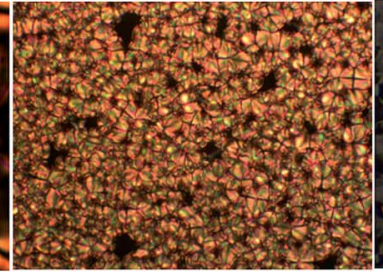

(b)

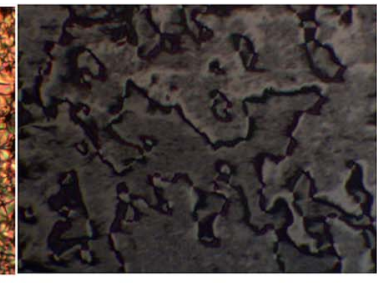

(c)

Fig. 4 The optical textures observed for the 1:2-BiPy/5OBA mixture: (a) the Schlieren nematic texture; (b) the focal conic fan texture with coexisting homeotropic regions seen for the smectic A phase; (c) the Schlieren smectic $C$ texture.

The trends in liquid crystalline behaviour revealed in Fig. 3 and 5 would most commonly be attributed to the formation of a $1: 2$ hydrogen bonded heterocomplex between the hydrogen bond donor, 5OBA, and the hydrogen bond acceptor, XBiPy, as shown schematically in Fig. 1(f). Within this explanation, the enhanced structural anisotropy of the heterocomplex compared to the structures of the individual components is used to account for the promotion of liquid crystallinity in the mixtures. In order to examine the validity of this interpretation, we now turn our attention to the transitional properties of the 1:2-XBiPy/5OBA mixtures and these are listed in Table 2 . As we have seen already in the phase diagrams shown in Fig. 3, the $1: 2$-EthaBiPy/5OBA mixture shows exclusively SmA behaviour, the $1: 2$-BiPy/5OBA, $1: 2$-EthylBiPy/5OBA and $1: 2$-ButBiPy/5OBA mixtures exhibit smectic A and nematic phases, whereas the $1: 2$-PropBiPy/5OBA mixture is not liquid crystalline.

The entropy changes associated with the clearing transitions, see Table 2, are entirely consistent with the proposed 


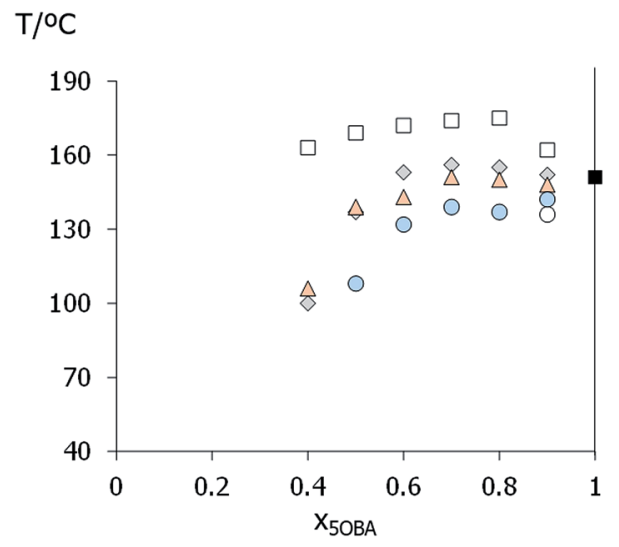

Fig. 5 Liquid crystal-isotropic transition temperature of the mixtures as a function of 5OBA mole fraction, $x_{50 B A}: \diamond B i P y / 5 O B A, \square$ EthylBipy/ 5OBA, $\triangle$ EthaBipy/5OBA, O PropBipy/5OBA; c ButBipy/5OBA. Also shown is the nematic to isotropic transition, $T_{\mathrm{N}-1}$, for $50 \mathrm{OA}$

phase assignments, ${ }^{46}$ although it may be argued that for ButBiPy/5OBA the entropy change is low if compared to those observed for even-membered liquid crystal dimers. ${ }^{47}$ At first sight, these thermodynamic data may be viewed as being broadly consistent with the essentially quantitative formation of 1:2-XBiPy/5OBA H-bonded complexes. However, a closer inspection reveals a number of significant inconsistencies with this intuitively pleasing explanation. First, the spread of transition temperatures between the proposed hydrogen bonded complexes is considerably narrower than that seen for covalently bound analogues. To illustrate this, Table 3 shows the transition temperatures for a set of low molar mass liquid crystals containing the same central linkage as found in the XBiPy hydrogen bond acceptors, but in which the hydrogen bonds have been replaced by Schiff's base linkages. ${ }^{48}$ In the covalent materials, replacing a butyl spacer by a single bond increases the nematic-isotropic transition temperature, $T_{\mathrm{N}-\mathrm{I}}$, by over $120{ }^{\circ} \mathrm{C}$. By comparison the same structural change in the 1:2-XBiPy/5OBA mixtures increases $T_{\mathrm{N}-\mathrm{I}}$ by only $17{ }^{\circ} \mathrm{C}$. An even larger difference is apparent on replacing an ethyl linkage by a single bond, namely over $78{ }^{\circ} \mathrm{C}$ in the covalent example and just $5{ }^{\circ} \mathrm{C}$ for the corresponding 1:2-XBiPy/5OBA mixtures.

The second inconsistency with the suggestion of the quantitative formation of the $1: 2$ complex involves the dependence of the clearing temperature on composition.
Table 3 Transition temperatures for the compounds ${ }^{48} a$

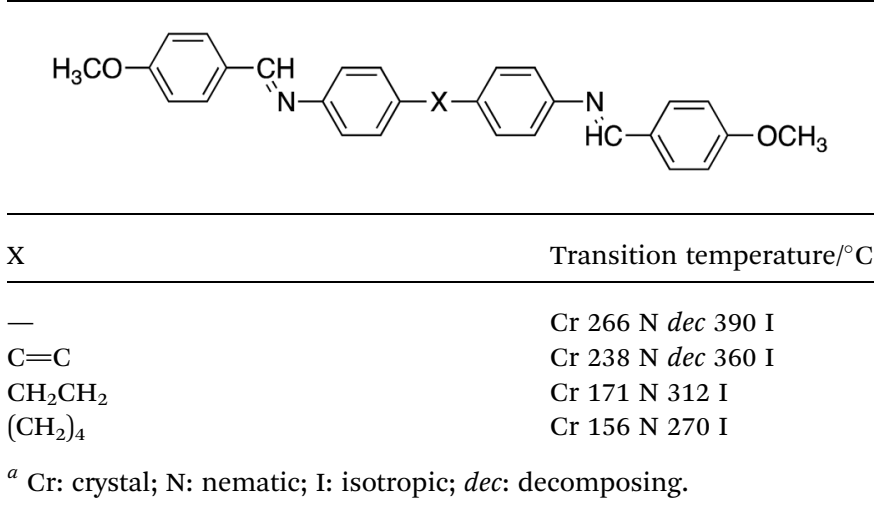

Specifically, the phase diagrams do not show pronounced maxima in the liquid-crystal-isotropic transition temperature curve for the corresponding 1:2-XBiPy/5OBA mixtures as would be expected if the hydrogen bonded complex was formed quantitatively. ${ }^{\mathbf{1 3}}$ Instead, the liquid crystal-isotropic transition curves are rather flat, see Fig. 3 and 5, and similar behaviour has been observed for other HBLCs. ${ }^{\mathbf{3 9 , 4 0 , 4 9}}$

Finally, the emergence of smectic behaviour seen for the mixtures is difficult to understand in terms of the proposed quantitative complexes given the solely nematogenic behaviour of 5OBA. The formation of smectic phases in conventional low molar mass mesogens is normally considered in terms of a microphase separation in which the mesogenic cores form one region while the alkyl chains constitute another. There are two major forces responsible for such a separation; energetically, the mean of the core-core and chain-chain interactions is more favourable than the mixed core-chain interaction, and entropically, the interaction between a core and a chain acts to order the chain and hence is unfavourable. Thus, the very general observation is that increasing the chain length in a given homologous series promotes smectic behaviour. ${ }^{50}$ On mixing 5OBA with BiPy, EthyBiPy, EthaBiPy, and ButBiPy however, we observe a very pronounced induction of smectic A behaviour in the 1:2 mixtures. This cannot be rationalised simply in terms of the formation of the $1: 2$ heterocomplex because the extension of the length of the semi-rigid core in the heterocomplex for the same terminal chain length compared with the 5OBA dimer would be expected to reduce stability of the smectic relative to the nematic phase, and quite

Table 2 Transitional properties of the 1:2-XBiPy/5OBA mixtures ( $\mathrm{Cr}$, crystal; SmA, smectic A; N, nematic; I, isotropic)

\begin{tabular}{|c|c|c|c|c|}
\hline & $T_{\mathrm{Cr}-\mathrm{SmA}} /{ }^{\circ} \mathrm{C},{ }^{\mathrm{a}} T_{\mathrm{SmA}-\mathrm{N}} /{ }^{\circ} \mathrm{C}$ & $T_{\mathrm{SmA}-\mathrm{I}} /{ }^{\circ} \mathrm{C},{ }^{\mathrm{b}} T_{\mathrm{N}-\mathrm{I}} /{ }^{\circ} \mathrm{C} ;{ }^{\mathrm{c}} T_{\mathrm{Cr}-\mathrm{I}} /{ }^{\circ} \mathrm{C}$ & $\Delta S_{\mathrm{Cr}-\mathrm{SmA}} / R,{ }^{\mathrm{a}} \Delta S_{\mathrm{SmA}-\mathrm{N}} / R$ & $\Delta S_{\mathrm{SmA-I}} / R,{ }^{\mathrm{b}} \Delta S_{\mathrm{N}-\mathrm{I}} / R$ \\
\hline BiPy/5OBA & $120,{ }^{a} 155$ & $\mathrm{~b}_{156}$ & $3.02,{ }^{\mathrm{a}} 0.61$ & $\mathrm{~b}_{0.55}$ \\
\hline EthylBiPy/5OBA & $128,{ }^{\mathrm{a}} 172$ & $\mathrm{~b}_{174}$ & $1.58,{ }^{\mathrm{a}} 0.54$ & ${ }^{\mathrm{b}} 0.57$ \\
\hline PropBiPy/5OBA & - & ${ }^{c} 95$ & - & - \\
\hline ButBiPy/5OBA & $121,{ }^{\mathrm{a}} 130$ & 139 & $1.49,{ }^{\mathrm{a}} 0.55$ & b 0.54 \\
\hline
\end{tabular}


the opposite is observed. We will return to these observations later.

\subsection{Temperature dependence of the FT-IR spectra of the 1:2- XBiPy/5OBA mixtures}

To more realistically interpret and account for the transitional behaviour of the 1:2-XBiPy/5OBA mixtures, a better understanding of the nature and extent of the hydrogen bonding within the system is required, and FT-IR is a particularly powerful tool for this purpose. Thus, Fig. 6 shows the temperature dependence of the high frequency region of the FT-IR spectra of the 1:2-XBiPy/5OBA mixtures, and these spectra share several common features. In each set of spectra, the high intensity $\mathrm{C}-\mathrm{H}$ stretching signals are superimposed on a broad band centred at $\nu \sim 2900 \mathrm{~cm}^{-1}$, associated with the fundamental vibration of hydrogen bonded hydroxyl groups, $\mathrm{OH}$, and referred to as the A-type band. ${ }^{\mathbf{8}, 51,52}$ Its appearance, together with the absence of contributions arising from free hydroxyl groups at $\nu>3500 \mathrm{~cm}^{-1}$, is indicative of extensive hydrogen bonding. ${ }^{53}$ This view is further supported by the observation of the B-type bands in the $2700-2500 \mathrm{~cm}^{-1}$ range, assigned to resonances between the fundamental stretch and in-plane bending of $\mathrm{OH}$ groups. The C-type band is also visible at around $1920 \mathrm{~cm}^{-1}$ and arises from the interaction between the fundamental stretching vibration and the first overtone of the torsional contributions of the $\mathrm{OH}$ groups. ${ }^{53,54}$

These bands exhibit their strongest values in the isotropic phase and, on cooling, they undergo step-reductions at the phase transitions. More specifically, the B-bands shown by the BiPy/5OBA and EthylBiPy/5OBA mixtures, Fig. 6(a) and (b), respectively, display strong and stable values within the smectic phase, whereas for EthaBiPy/5OBA and ButBiPy/5OBA, Fig. 6(c) and (e), respectively, they decrease markedly in intensity on cooling within the liquid crystal ranges. The
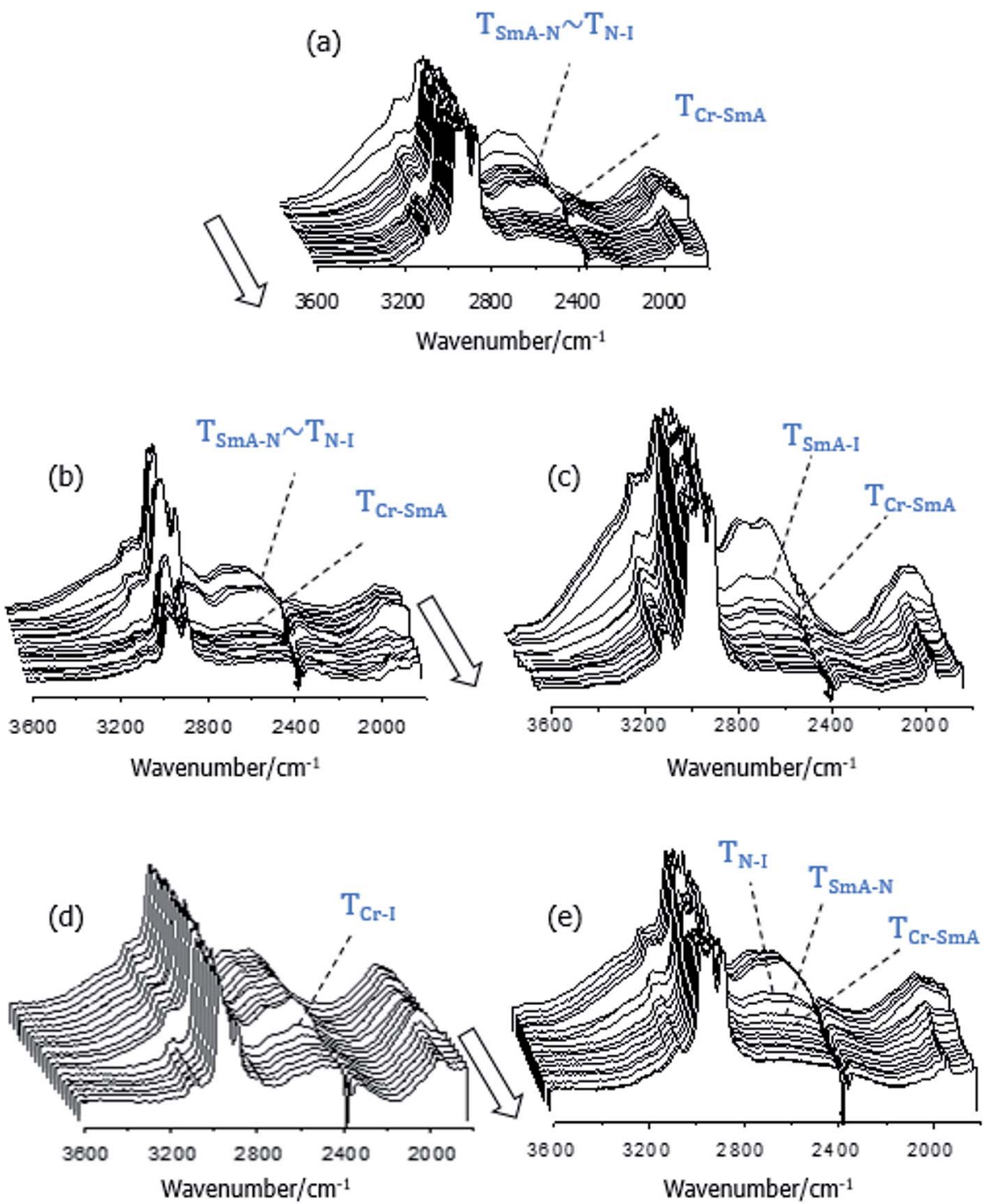

Fig. 6 Temperature dependence of the high frequency region of the FT-IR spectra for the $1: 2$ mixtures of: (a) BiPy/5OBA, (b) EthylBiPy/5OBA, (c) EthaBiPy/5OBA, (d) PropBiPy/5OBA, and (e) ButBiPy/5OBA. Arrows indicate the direction of cooling. 
Fermi bands of all the samples, including PropBiPy/5OBA, Fig. 6(d), show a pronounced decrease in intensity on crystallisation.

The reduction in intensity of the $\mathrm{OH}$ Fermi bands on cooling may at first sight appear counter intuitive given the expectation of the enhanced formation of hydrogen bonds at lower temperatures. The considerable reduction in intensity observed in these and other IR bands in the spectra on cooling may be attributed to the strong tendency of hydrogen bonded liquid crystals to spontaneously align on polar surfaces. ${ }^{55-58}$ Such director alignment will have a particularly marked effect on bands associated either parallel or perpendicular to the molecular long axis, and may also affect the crystallisation process as appears to be the case for the XBiPy/5OBA mixtures.

The suggestion that some of the acid groups may be interacting preferentially with the surfaces implies that the heterocomplex cannot be formed quantitatively. This view is further supported by the shape of the Fermi bands seen in Fig. 6. In strongly hydrogen bonded heterocomplexes, we would expect the C-band to be more pronounced with respect to the A-band, resulting in a smoother profile of the B-region
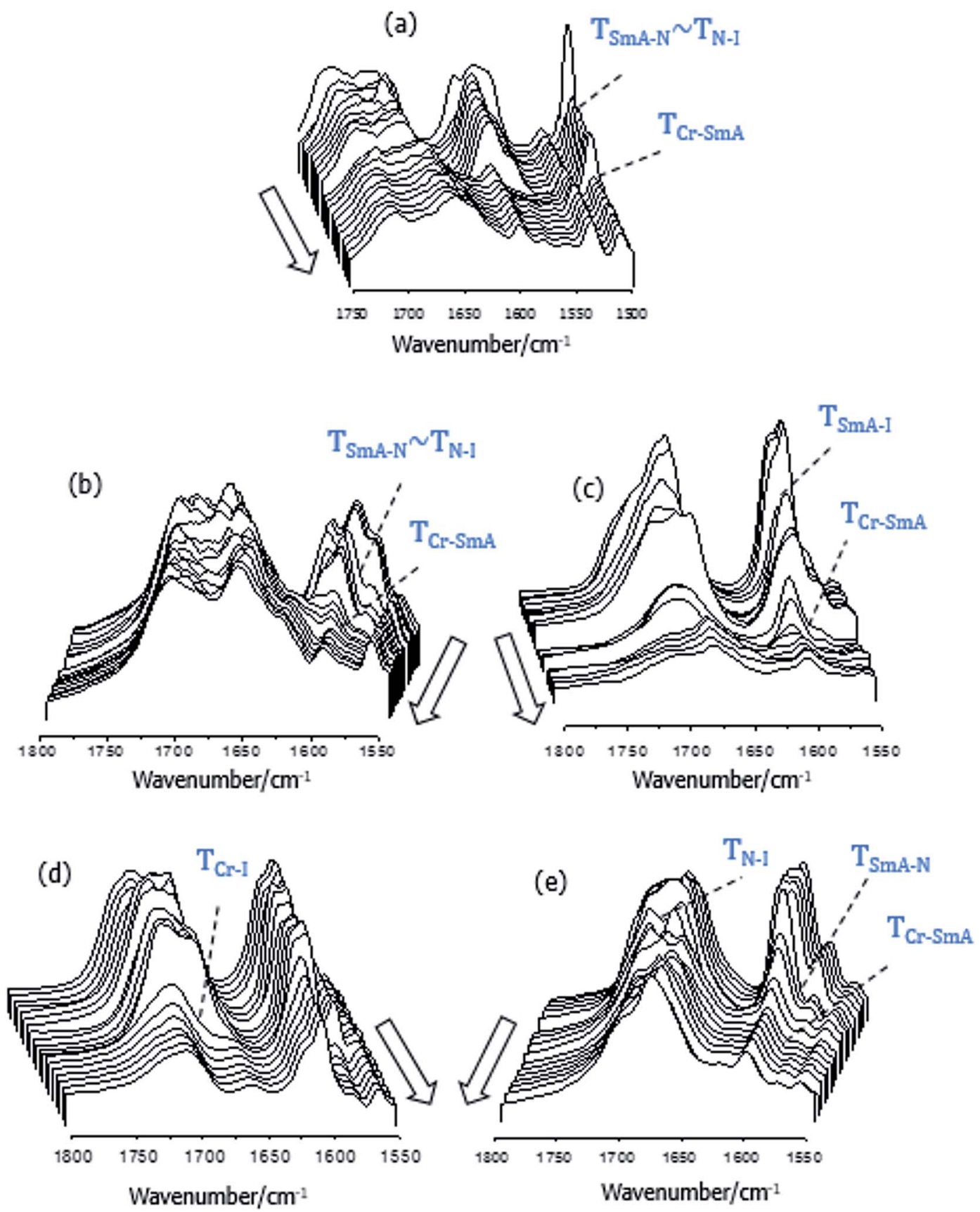

Fig. 7 Temperature dependence of the carbonyl and aromatic stretching regions of the FT-IR spectra for the $1: 2$ mixtures of: (a) BiPy/5OBA, (b) EthylBiPy/5OBA, (c) EthaBiPy/5OBA, (d) PropBiPy/5OBA, and (e) ButBiPy/5OBA. Arrows indicate the direction of cooling. 
which is shifted to lower frequencies. ${ }^{\mathbf{5 4 , 5 9 , 6 0}}$ However, this is not the case for the spectra of the 1:2-XBiPy/5OBA mixtures in which the B-band still displays two maxima, suggesting the presence of rather weak hydrogen bonds such as those displayed by, for example, 5OBA. ${ }^{52}$

If we now turn our attention to the carbonyl stretching region of the FT-IR spectra of the 1:2-XBiPy/5OBA mixtures shown in Fig. 7, we see a rather complex profile indicating that the $\mathrm{C}=\mathrm{O}$ groups are found in a broad range of chemical environments. This supports the view that the heterocomplexes are not formed quantitatively, but instead coexist in equilibria with other species, namely, cyclic and open acid dimers, free acids and, necessarily, free bipyridines, XBiPy, see Fig. $8 .^{38-40}$ The strong reductions in intensity taking place at the phase transitions are, at least in part, a result of the spontaneous director alignment described earlier, and this also effects the intensities of the aromatic ring vibrations, $\nu \sim$ 1600-1500 $\mathrm{cm}^{-1}$. In order to assess the individual carbonyl contributions arising from the species shown in Fig. 8, the 1750-1650 $\mathrm{cm}^{-1}$ region has been fitted using a sum of Gaussian peaks, see Fig. 9. The band arising from the heterocomplexes, $\mathrm{C}=\mathrm{O}_{\text {het }}$, is expected at $\sim 1700 \mathrm{~cm}^{-1},{ }^{61}$ together with signals corresponding to monomeric (free) acids, $\mathrm{C}=$ $\mathrm{O}_{\text {mon }}$ at $\nu \geq 1730 \mathrm{~cm}^{-1}$; open dimers, $\mathrm{C}=\mathrm{O}_{\text {op }}$ at $\nu \sim 1730-1680$ $\mathrm{cm}^{-1}$, closed dimers, $\mathrm{C}=\mathrm{O}_{\mathrm{cl}}$ at $\nu \sim 1680 \mathrm{~cm}^{-1}$, and chain-like catemer aggregates, $\mathrm{C}=\mathrm{O}_{\text {cat }}$ at $\nu \leq 1660 \mathrm{~cm}^{-1} \cdot{ }^{52,62}$ In addition, we have split the $\mathrm{C}=\mathrm{O}_{\mathrm{op}}$ contribution into two identical but shifted bands to account for the two types of $\mathrm{C}=\mathrm{O}$ groups expected in the open dimers: specifically, those participating as hydrogen bond acceptors, $\mathrm{C}=\mathrm{O}_{\text {opLow }}$, or

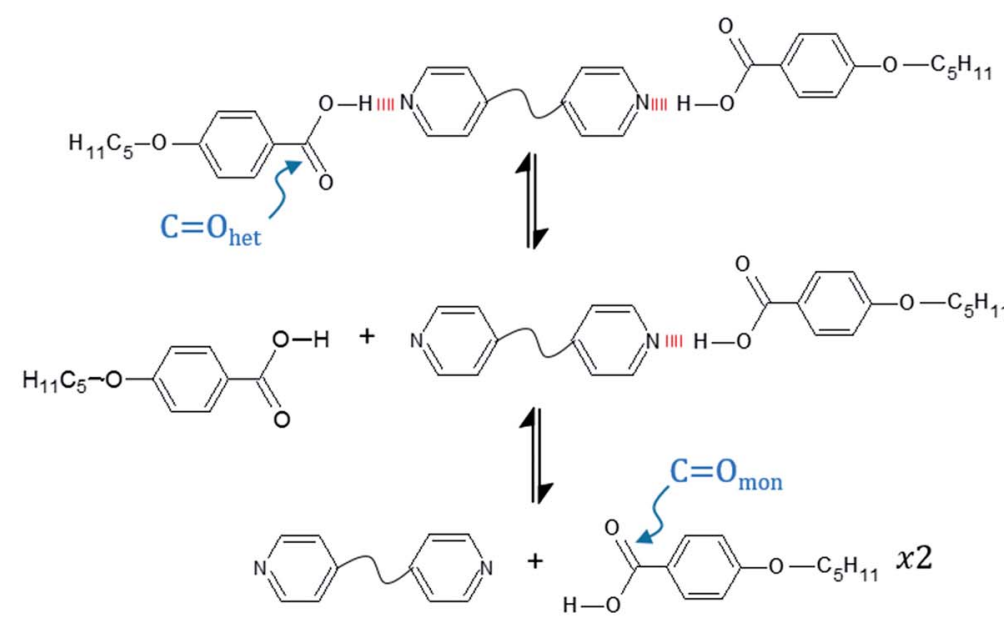

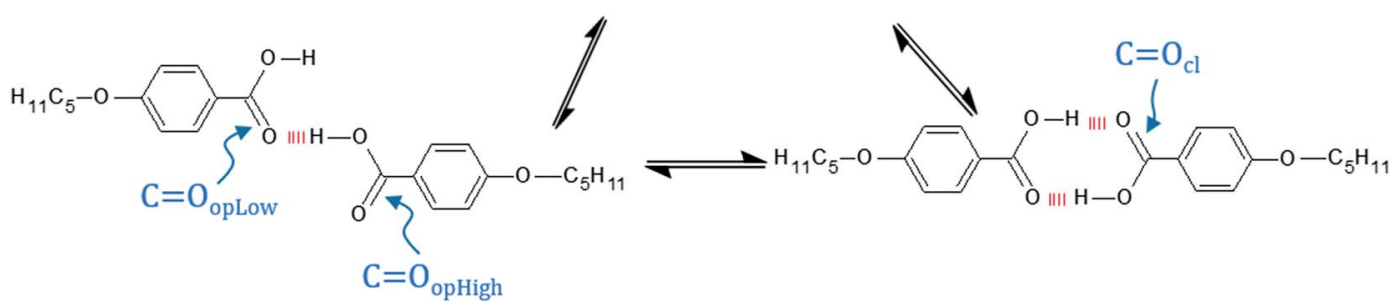

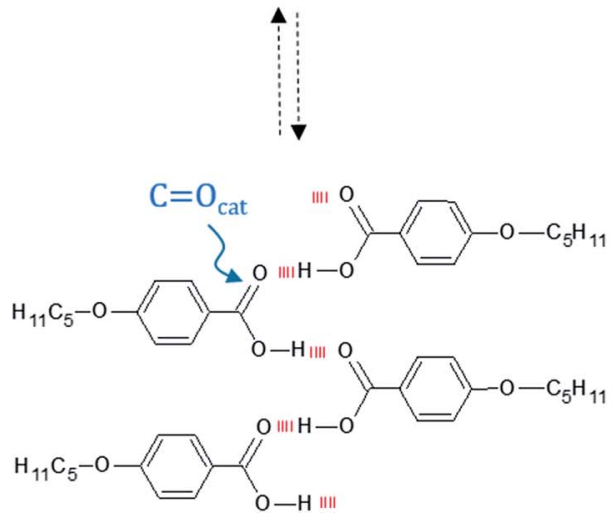

Fig. 8 Sketches of the possible species present in the 1:2-XBiPy/5OBA mixtures and assignment of the carbonyl stretches ( $\mathrm{C}=\mathrm{O}$ ). Heterocomplexes, $\mathrm{C}=\mathrm{O}_{\text {het }}$; monomeric species, $\mathrm{C}=\mathrm{O}_{\text {mon }}$ (free acids); closed dimers, $\mathrm{C}=\mathrm{O}_{\mathrm{cl}}$; carbonyl groups attached to $\mathrm{OH}$ groups hydrogen bonded in open dimers, $\mathrm{C}=\mathrm{O}_{\mathrm{opHigh}}$; carbonyl groups hydrogen bonded in open dimers, $\mathrm{C}=\mathrm{O}_{\mathrm{opLow}}$; catemer-like aggregates, $\mathrm{C}=\mathrm{O}_{\text {cat }}$. 
those attached to the $\mathrm{O}-\mathrm{H}$ group that is hydrogen bonded, $\mathrm{C}=$ $\mathrm{O}_{\text {opHigh }}{ }^{10}$

We have performed this fitting procedure for the carbonyl region for all the spectra obtained as a function of temperature for each mixture, and used the relative areas of each of the Gaussian fitting peaks, $\mathrm{A}\left[\mathrm{C}=\mathrm{O}_{\mathrm{i}}\right]$, to the total scaled band area as a measure of the relative concentration of that particular species, see Fig. 10. In doing this we scale the peak areas relative to the number of carbonyl groups in each species. Thus, the areas obtained from the free acid contribution, $\mathrm{A}\left[\mathrm{C}=\mathrm{O}_{\mathrm{mon}}\right]$, are assumed to be directly associated with the concentration of free acid. However, the relative amounts of the heterocomplex and dimeric acid species have been estimated by dividing the corresponding peak areas by 2 , i.e., $\mathrm{A}\left[\mathrm{C}=\mathrm{O}_{\text {het }}\right] / 2, \mathrm{~A}\left[\mathrm{C}=\mathrm{O}_{\mathrm{cl}}\right] / 2$, and $\mathrm{A}\left[\mathrm{C}=\mathrm{O}_{\mathrm{op}}\right] / 2$. In this analysis we have not explicitly accounted for the possible formation of $1: 1$ heterocomplexes shown in Fig. 8. Furthermore, we have not scaled the peak areas associated with the catemeric species and note that given these represent strands of acid molecules, we are over estimating their relative concentration in this analysis.

The space filling model, electrostatic potential isosurface and dipole moment calculated for the open and closed dimers of 5OBA are shown in Fig. 11. The closed dimer is energetically more favourable by $40.62 \mathrm{~kJ} \mathrm{~mol}^{-1}$. It is interesting to note that in the open dimer a strained six-membered ring is formed and this has not previously been suggested in schematic representations of this complex. The space filling model, electrostatic potential isosurface and dipole moment calculated for each 1:2-XBiPy/5OBA heterocomplex are shown in Fig. 12, and the estimated hydrogen bond dissociation energies are listed in Table 4. The larger dipole moment calculated for the

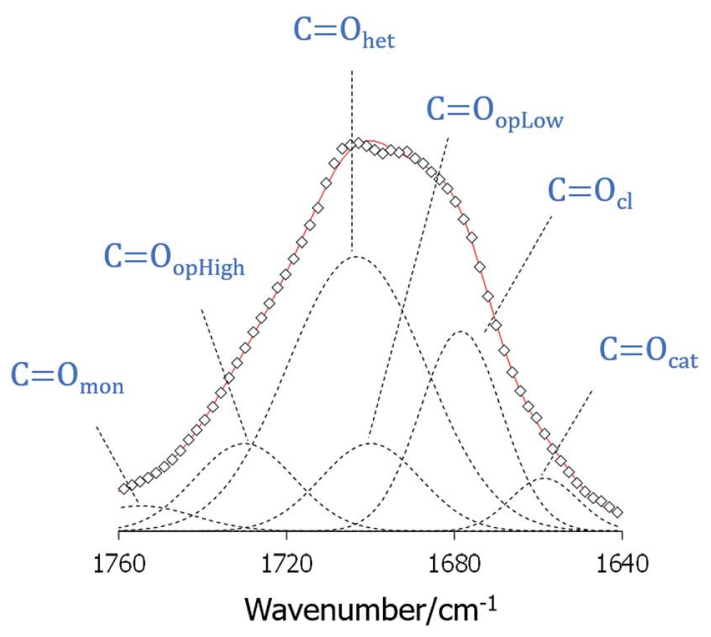

Fig. 9 Assignment of individual species' contributions to the $\mathrm{C}=\mathrm{O}$ stretching region of the FT-IR spectrum of $1: 2-B u t B i P y / 5 O B A$ measured in the isotropic melt. From left to right: monomeric species, $\mathrm{C}=\mathrm{O}_{\text {mon }}$ (free acids); open dimers where the $\mathrm{C}=\mathrm{O}$ group is not acting as hydrogen bond acceptor, $\mathrm{C}=\mathrm{O}_{\text {opHighi hetero- }}$ complexes, open dimers where the $\mathrm{C}=\mathrm{O}$ group acts as hydrogen bond acceptor, $\mathrm{C}=\mathrm{O}_{\text {oplowi }}$ closed dimers, $\mathrm{C}=\mathrm{O}_{\mathrm{cl}}$; catemer-like aggregates, $\mathrm{C}=\mathrm{O}_{\text {cat }}$.
$1: 2$-PropBiPy/5OBA complex arises from its bent shape and we will discuss this later. The H-bond dissociation energy calculated for the closed dimer is approximately twice that of the open dimer, and this simply reflects that in the former there are two identical hydrogen bonds and in the latter two longer and different hydrogen bonds. The hydrogen bond dissociation energies of the heterocomplexes are similar and larger than that calculated for the closed dimer formed by 5OBA.

According to the data shown in Fig. 10, the heterocomplex tends to be the most prevalent species in each mixture and particularly at higher temperatures. This is consistent with the higher strength of the mixed Pyr $\cdots$ HO hydrogen bond with respect to the acid-acid hydrogen bond listed in Table 4 . It is immediately apparent, however, that for all the mixtures other species are present in significant amounts over the whole temperature range studied. The variation in the concentrations of the species present are complex and change from mixture to mixture. In general, however, the concentration of closed dimers tends to increase on cooling while that of the open dimers decreases. This observation is consistent with the shape and position of the B-bands in the spectra shown in Fig. 6. In the case of 1:2-EthaBiPy/5OBA, essentially similar amounts of the heterocomplex, closed and open acid dimers and catemeric strands are seen in the smectic phase. The concentration of free acid tends to be very small in each mixture, except in the crystal phase of 1:2PropBiPy/5OBA.

An increase in the concentration of catemer-like aggregates tends to be associated with crystallisation, and this has been related to the opening, and linking of closed dimers within a smectic structure. ${ }^{10}$ Significant concentrations of open dimers are seen in the mixtures, especially in the isotropic range. We note that the samples showing the highest transition temperatures, BiPy/5OBA and EthylBiPy/5OBA, have the largest amounts of open dimers in the smectic phase, see Fig. 10(a) and (b), and we will return to this observation later.

Finally, the behaviour of the non-mesogenic 1:2-PropBiPy/ 5OBA mixture appears to be qualitatively different. In addition to the increase in free acid concentration on crystallisation, noted earlier, this mixture also exhibits the highest concentration of the heterocomplex, particularly in the crystal phase, coinciding with low concentrations of acid dimers.

\section{Concluding remarks}

The FT-IR spectra of the XBiPy/5OBA mixtures show clearly that a number of differing species exist in equilibria over the temperature range studied. Furthermore, although the concentration of the heterocomplex tends to be the greatest at a given temperature, presumably reflecting the higher strength of the mixed hydrogen bond, see Table 4, very significant amounts of other species are also always present. Thus, the phase behaviour of these mixtures should not be interpreted simply in terms of the structural anisotropy of the 


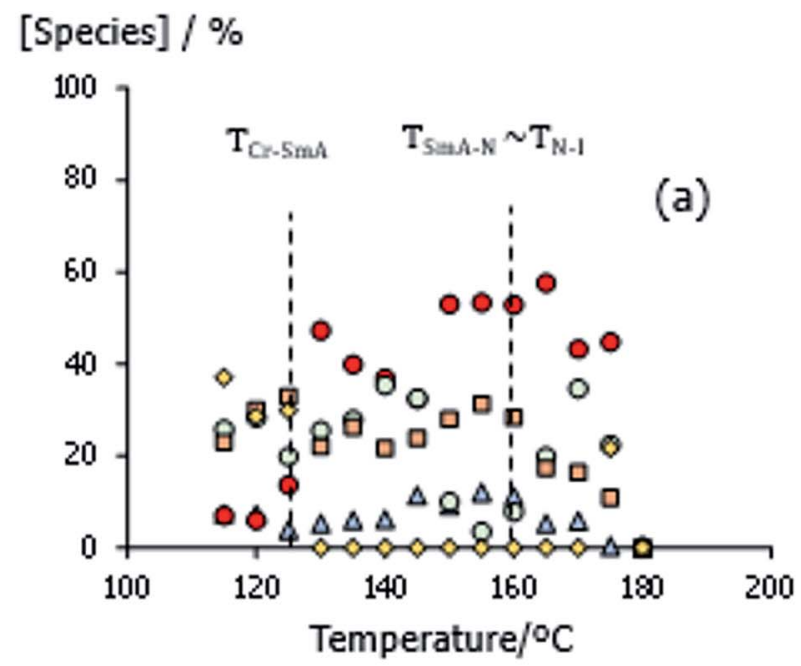

[Species] /\%

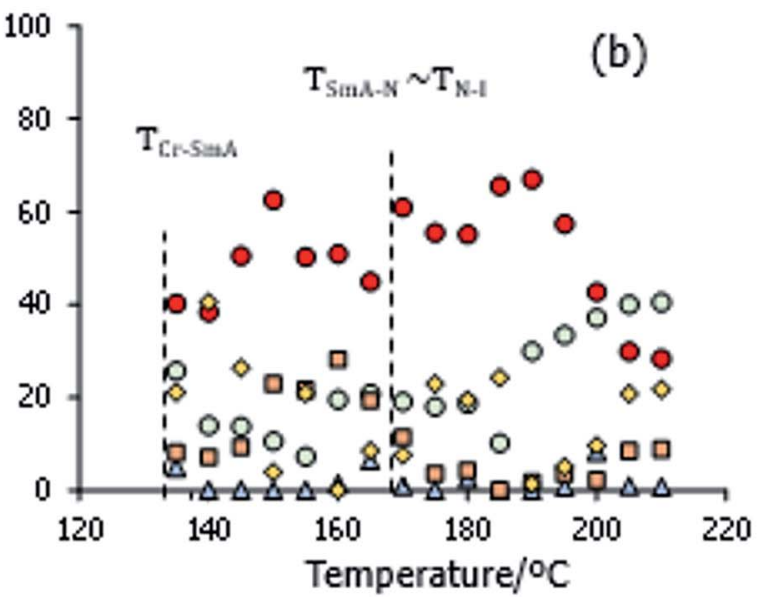

[Species] /\%

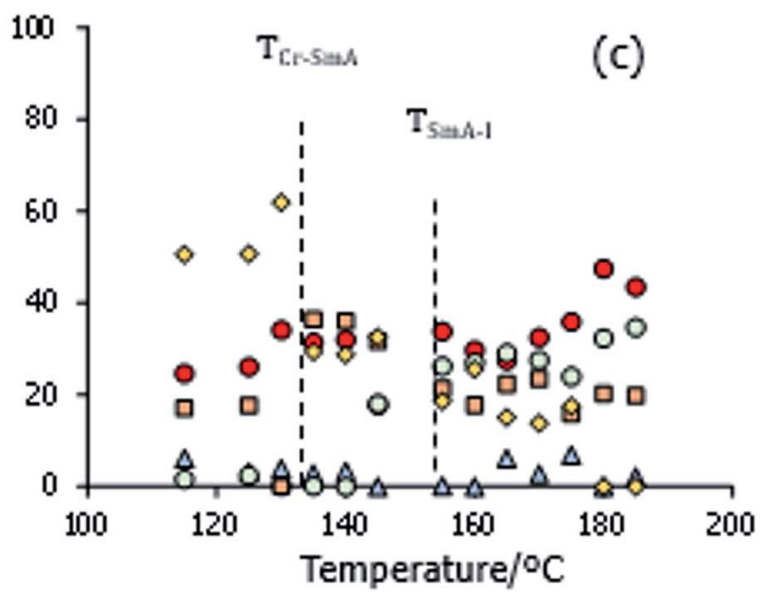

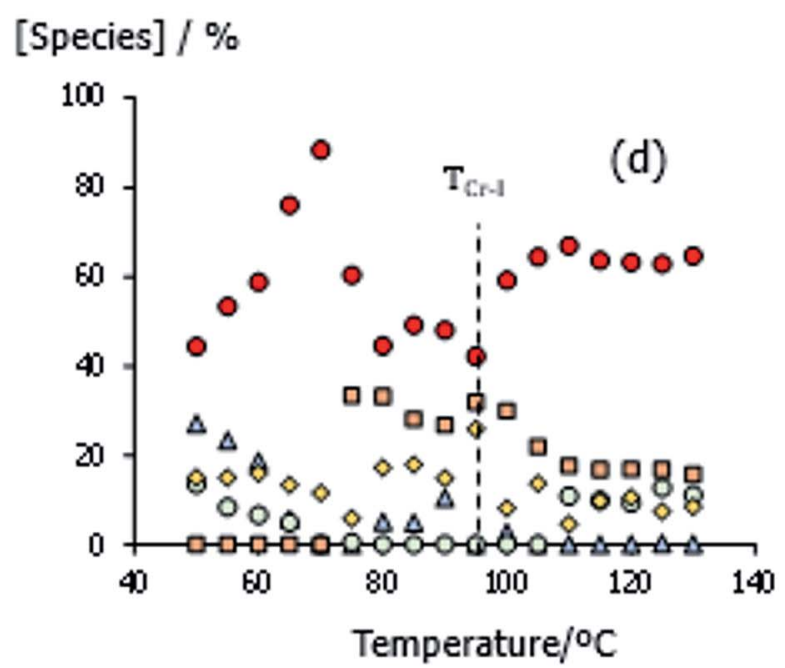

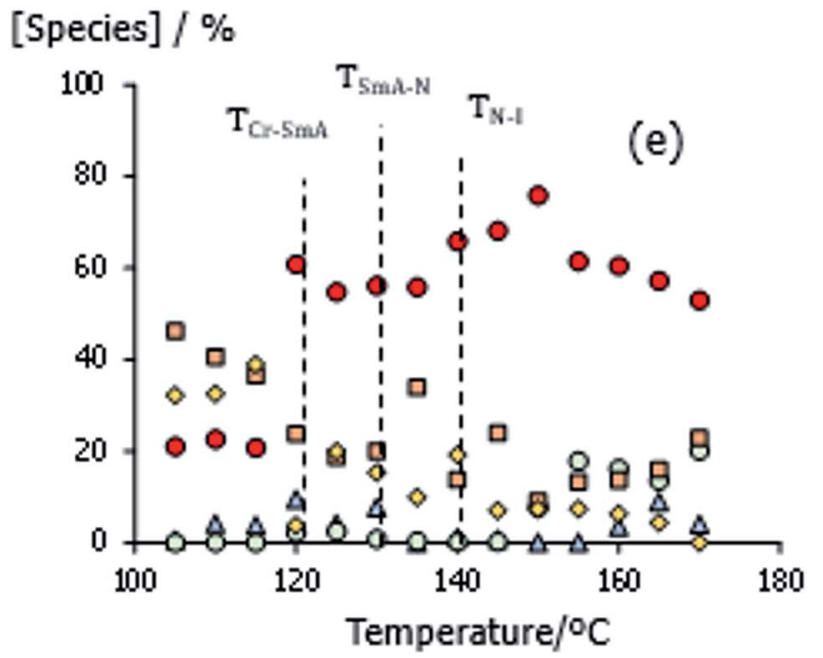

Fig. 10 Temperature dependence of the estimated concentrations of the species in the $1: 2$ mixtures, based on the scaled relative areas of the $\mathrm{C}=\mathrm{O}$ individual contributions, $\mathrm{A}\left[\mathrm{C}=\mathrm{O}_{\mathrm{i}}\right]$ : (a) BiPy/5OBA, (b) EthylBiPy/5OBA, (c) EthaBiPy/5OBA, (d) PropBiPy/5OBA, and (e) ButBiPy/5OBA. heterocomplexes, $A\left[C=\mathrm{O}_{\text {het }}\right] / 2$, $\square$ closed dimers, $A\left[C=\mathrm{O}_{\mathrm{cl}}\right] / 2 ; \bigcirc$ open dimers, $\mathrm{A}\left[\mathrm{C}=\mathrm{O}_{\mathrm{op}}\right] / 2 ; \triangle$ monomeric species $\mathrm{A}\left[\mathrm{C}=\mathrm{O}_{\mathrm{mon}}\right] ; \diamond \mathrm{catemer}$ or chain aggregates, $\mathrm{A}\left[\mathrm{C}=\mathrm{O}_{\mathrm{cat}}\right]$. $T_{\mathrm{N}-1}$, nematic to isotropic transition; $T_{\mathrm{Cr}-\mathrm{SmA}}$, crystal to smectic $\mathrm{A}$ transition; $T_{\mathrm{SmA}-\mathrm{N}}$, smectic $A$ to nematic transition; $T_{\text {SmA-1, }}$ smectic A to isotropic transition; $T_{\mathrm{Cr}-1}$, crystal to isotropic transition. 


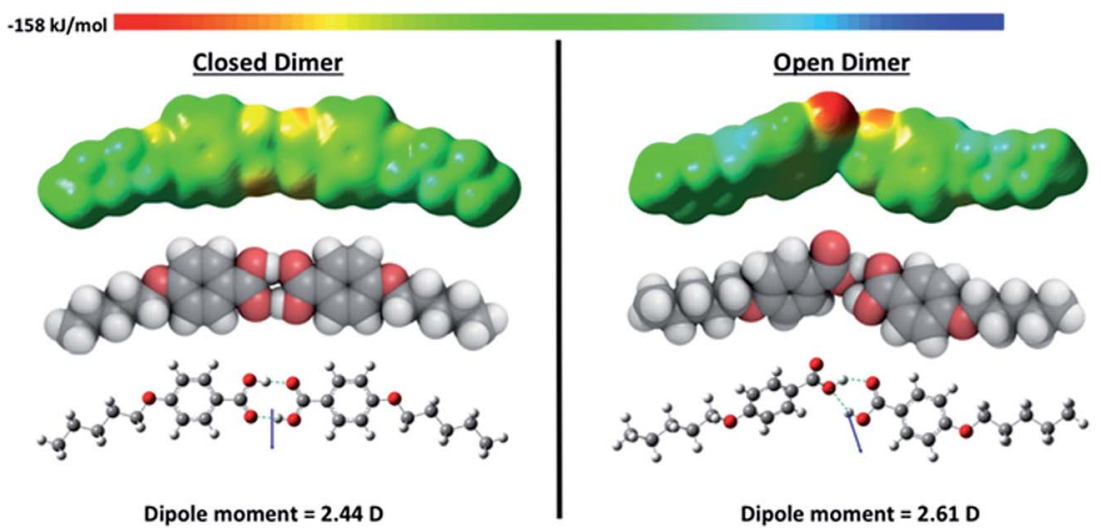

Fig. 11 The electrostatic potential isosurface (top), space filling (middle), and ball and stick models showing the dipole moment (bottom) for the closed and open dimers of 5OBA.

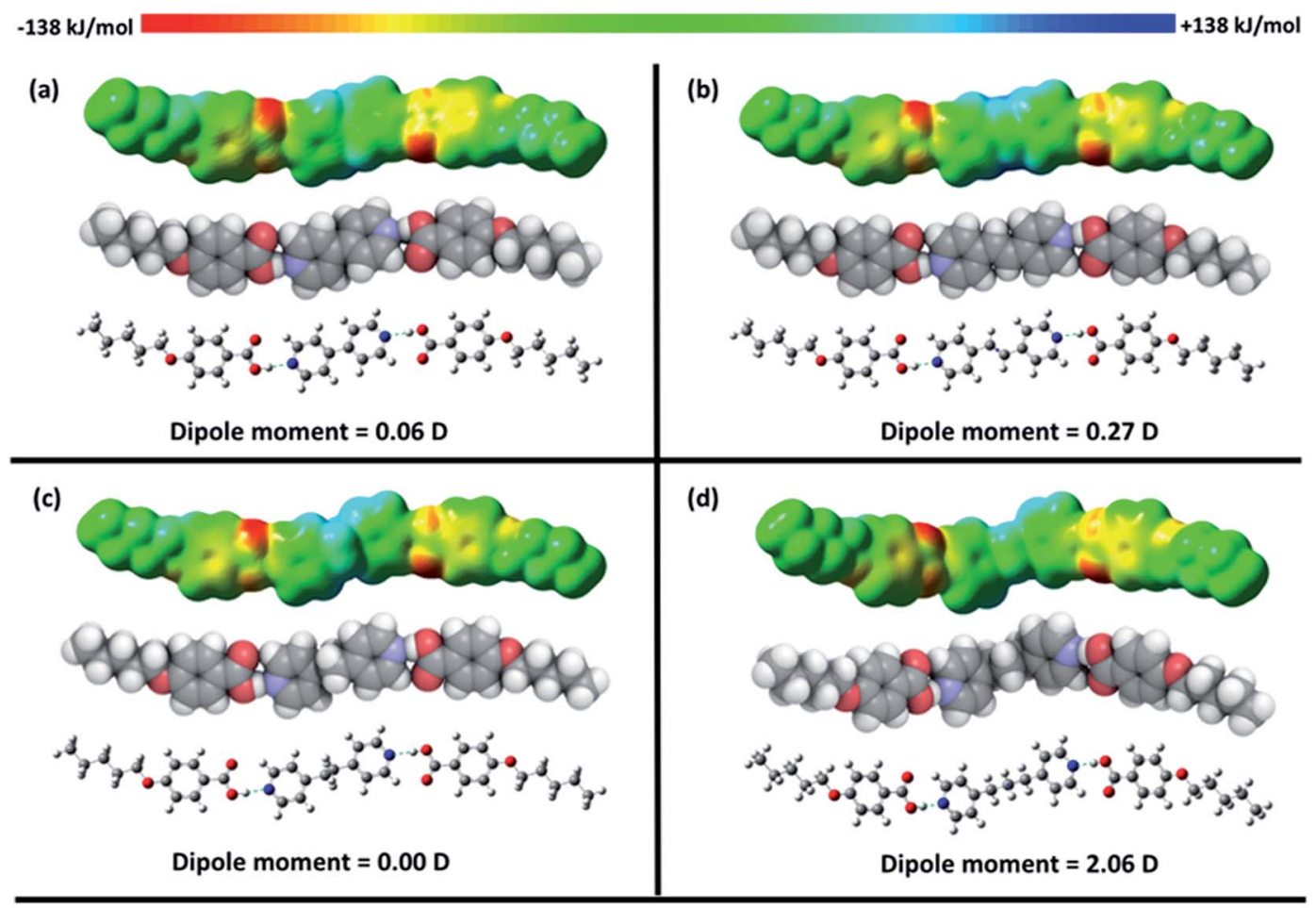

(e)

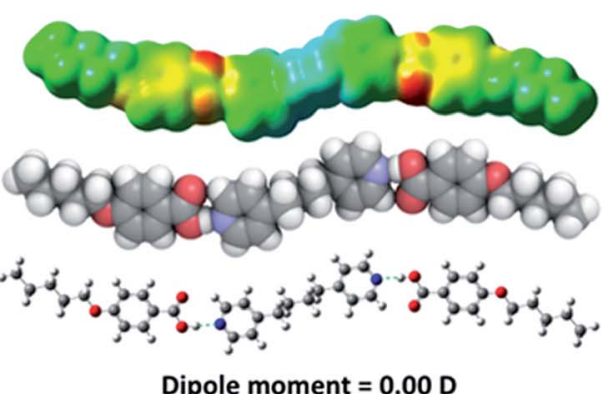

Fig. 12 The electrostatic potential isosurface (top), space filling (middle), and ball and stick models showing the dipole moment (bottom) for the $1: 2$ heterocomplexes formed by: (a) BiPy/5OBA, (b) EthylBiPy/5OBA, (c) EthaBiPy/5OBA, (d) PropBiPy/5OBA, and (e) ButBiPy/5OBA. 
Table 4 Hydrogen bond dissociation energies estimated for the structures shown in Fig. 11 and 12

\begin{tabular}{|c|c|c|c|}
\hline $\begin{array}{l}1: 2 \\
\text { Complex }\end{array}$ & $\begin{array}{l}\text { Average } \mathrm{H} \text { atom - } \\
\mathrm{H} \text {-bond acceptor } \\
\text { length in optimised } \\
\text { structure } / \AA\end{array}$ & $\begin{array}{l}\text { Average } \mathrm{H} \text { atom - } \\
\mathrm{H} \text { bond acceptor } \\
\text { length in separated } \\
\text { structure/̊ }\end{array}$ & $\begin{array}{l}\text { H-bond } \\
\text { dissociation } \\
\text { energy/ } \\
\mathrm{kJ} \mathrm{mol}^{-1}\end{array}$ \\
\hline $\begin{array}{l}\text { 5OBA closed } \\
\text { dimer }\end{array}$ & 1.672 & 4.998 & 96.06 \\
\hline 5OBA open & 1.833 & 5.000 & 47.29 \\
\hline dimer & 1.933 & 4.739 & \\
\hline BiPy/5OBA & 1.797 & 5.000 & 111.71 \\
\hline EthylBiPy/5OBA & 1.788 & 5.000 & 113.52 \\
\hline EthaBiPy/5OBA & 1.781 & 5.000 & 115.33 \\
\hline PropBiPy/5OBA & 1.778 & 5.000 & 116.14 \\
\hline $\begin{array}{l}\text { ButBiPy/ } \\
5 \text { OBA }\end{array}$ & 1.774 & 5.000 & 116.87 \\
\hline
\end{tabular}
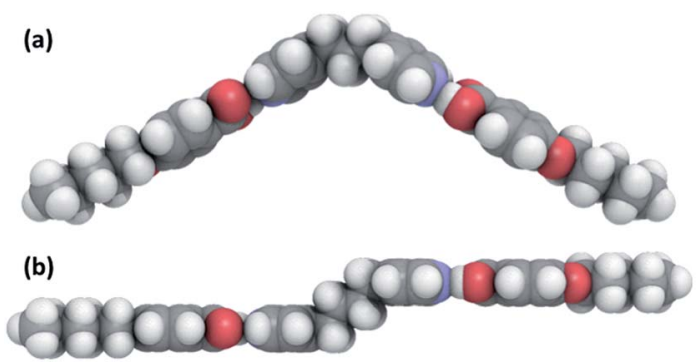

Fig. 13 Space filling models of the $1: 2$ heterocomplexes formed by (a) PropBiPy/5OBA and (b) ButBiPy/5OBA. heterocomplex. Instead we must also consider the impact of the acid dimers, free acid and XBiPy on phase behaviour.

We have seen that the trend in the clearing temperatures of the mixtures are in accord with predictions based on comparing the structure of the corresponding heterocomplexes with those of conventional covalent low mass liquid crystals. Thus, the highest clearing temperature is observed for the mixture in which the heterocomplex possesses the most linear, rigid core, namely, EthylBiPy/5OBA, see Fig. 12, and the lowest for the PropBiPy/5OBA mixture in which the heterocomplex has a nonlinear and flexible core which in fact does not show liquid crystalline behaviour. This bent shape is not immediately apparent in the perspectives shown in Fig. 12, but is highlighted in Fig. 13 which compares the molecular shapes of the $1: 2$ PropBiPy/5OBA and ButBiPy/5OBA heterocomplexes.

We noted that the spread of clearing temperatures exhibited by these mixtures is much narrower than that seen for analogous covalent low molar mass liquid crystals. Presumably this reflects the high concentrations of the other species present, and in particular of the acid dimers which are common to all the mixtures. The free acid and XBiPy components do not exhibit liquid crystallinity and so will reduce the clearing temperatures of the mixtures. The absence of liquid crystalline behaviour for the PropBiPy/5OBA mixture may now be attributed, not only to the shape of the heterocomplex, but also to that of the uncomplexed PropBiPy molecules which will strongly inhibit the formation of liquid crystal phases. The rather low entropy change associated with the nematicisotropic transition seen for the ButBiPy/5OBA mixture when compared to those observed for even-membered liquid crystal dimers ${ }^{63-66}$ is also readily explained in terms of the mixture of various hydrogen bonded and free species.

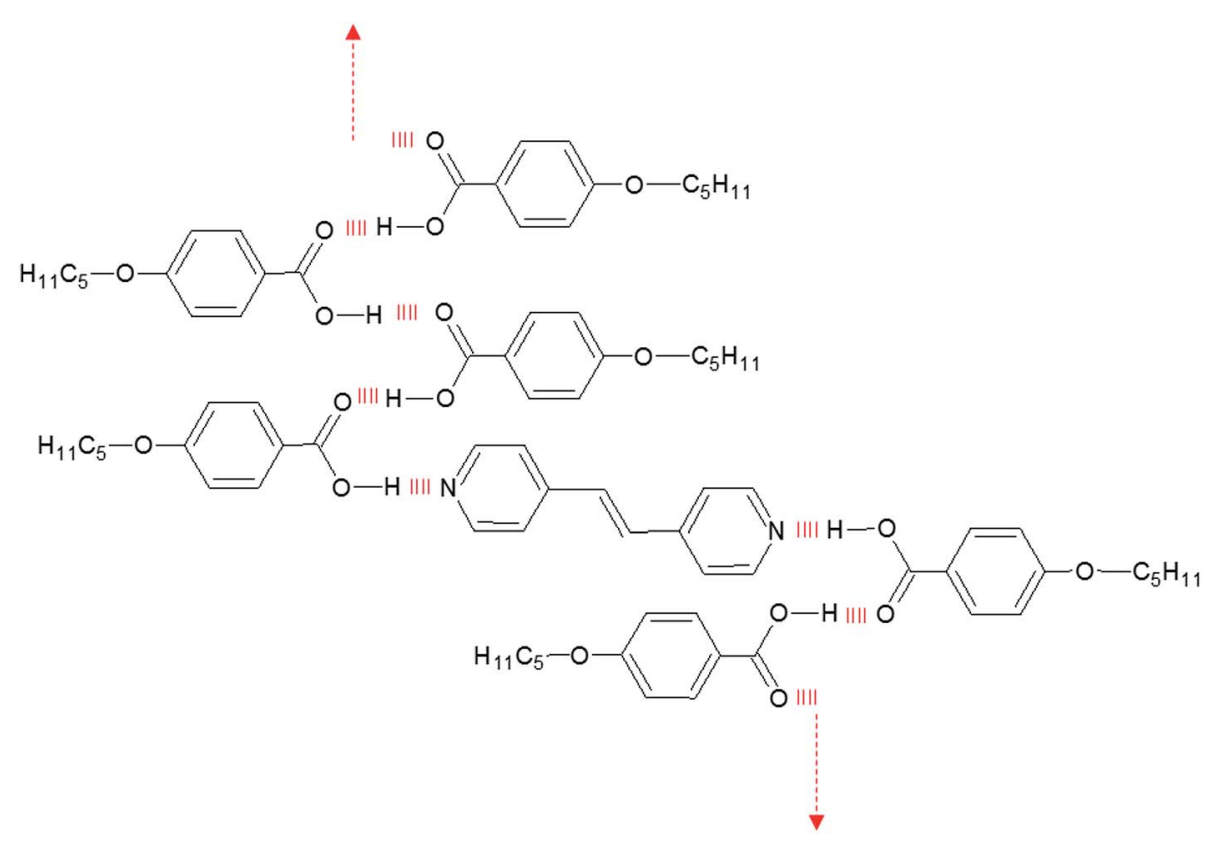

Fig. 14 Schematic representation of the stabilisation of the smectic phase in XBiPy/5OBA mixtures by lateral hydrogen bonding giving rise to catemeric-strands. 
We also noted earlier that the shapes of the phase diagrams seen in Fig. 3 were also inconsistent with the assumption of the quantitative formation of the heterocomplex and that the clearing temperature was rather insensitive to the composition of the mixture over a rather broad range. This may now be accounted for in terms of the complex equilibria between a number of differing species.

The third inconsistency in attempting to account for the liquid crystalline behaviour of these systems in terms of the quantitative formation of the heterocomplex involved the strong induction of smectic behaviour seen for the mixtures. However, the presence of open dimers and catemer-like strands, see Fig. 8, stabilises the layered arrangements and hence, strongly promotes smectic phases, ${ }^{67,68}$ see Fig. 14 . In a similar vein, it has been suggested recently that such lateral interactions may play an important role in stabilising the twist-bend nematic phase shown by 4 -[6-( $4^{\prime \prime}$-cyanobiphenyl-4-yl)hexyloxy]benzoic acid, CB6OBA, ${ }^{37}$ and the appearance of a cybotactic nematic phase that nucleates the smectic $\mathrm{C}$ domains of some 4-alkoxybenzoic acids. ${ }^{9,10,62}$ This is another manifestation of the "softness" of the hydrogen bond, and by extension of the supramolecular core, that allows for coexisting different configurations of supramolecular liquid crystals which stabilise the liquid crystal phase.

Our interpretation of the carbonyl stretching region of the FTIR spectra of the $\mathrm{XBiPy} / 5 \mathrm{OBA}$ mixtures in terms of various hydrogen-bonded and free species has allowed us to account for the liquid crystalline behaviour of these systems. Similar approaches described in earlier studies have involved acid dimers and heterocomplexes, ${ }^{\mathbf{3 8 - 4 0}}$ but to the best of our knowledge this is the first attempt to specifically include both carbonyl stretches associated with open acid dimers and has not had to assume the double minimum potential energy surface most commonly used to describe the unionised hydrogen bond between an acid and pyridyl fragments. ${ }^{59,61}$ We have also shown how lateral hydrogen bond interactions may give rise to catemer strands which in turn can stabilise smectic phases. This study now opens up new lines of investigation to relate the concentration fluctuations of these various supramolecular species to liquid crystal behaviour in symmetric and non-symmetric HBLCs, and how the lateral interactions may be utilised to tailor the structures obtained.

\section{References}

1 T. Kato and Y. Kamikawa, in Handbook of Liquid Crystals, ed. J. W. Goodby, P. J. Collings, T. Kato, C. Tschierske, H. F. Gleeson and P. Raynes, Wiley-VCH, Weinheim, Germany, 2nd edn, 2014, vol. 5, pp. 513-540.

2 C. M. Paleos and D. Tsiourvas, Supramolecular hydrogenbonded liquid crystals, Liq. Cryst., 2001, 28(8), 11271161.

3 G. W. Gray and B. Jones, Mesomorphism and chemical constitution 3. The effect of halogen substitution on the mesomorphism of the 4-alkoxybenzoic acids, J. Chem. Soc., 1954, 2556-2562.

4 G. W. Gray and B. Jones, The mesomorphic transition points of the para-normal-alkoxybenzoic acids - a correction, $J$. Chem. Soc., 1953, 4179-4180.
5 G. W. Gray and B. Jones, Influence of substituents on the mesomorphism of para-normal-alkoxybenzoic and 6normal-alkoxy-2-naphthoic acids, Nature, 1952, 170(4324), 451-452.

6 S. S. Sastry, K. L. Sarada, K. Mallika, C. N. Rao, S. Lakhminarayana and H. S. Tiong, Eigen value analysis studies on hydrogen-bonded mesogens, Liq. Cryst., 2014, 41(10), 1483-1494.

7 A. V. N. A. Kumar, B. Sridevi, M. Srinivasulu, P. V. Chalapathi and D. M. Potukuchi, Inductive effect for the phase stability in hydrogen bonded liquid crystals, x-(p/m)BA:9OBAs, Liq. Cryst., 2014, 41(2), 184-196.

8 A. Azima, C. W. Brown and S. S. Mitra, Temperaturedependence of IR-spectra of liquid-crystalline $p$ heptoxybenzoic acid and p-octoxybenzoic acid, Spectrochim. Acta, Part A, 1975, 31(9-10), 1475-1479.

9 S. C. Ilyin and I. I. Konstantinov, Rheological evidence for the existence of subphases in the liquid crystalline 4-nalkoxybenzoic acids, Liq. Cryst., 2016, 43(3), 369-380.

10 M. J. Abdy, A. Murdoch and A. Martinez-Felipe, New insights into the role of hydrogen bonding on the liquid crystal behaviour of 4-alkoxybenzoic acids: a detailed IR spectroscopy study, Liq. Cryst., 2016, DOI: 10.1080/ 02678292.2016.1212119.

11 T. Kato and J. M. J. Frechet, New approach to mesophase stabilization through hydrogen-bonding molecularinteractions in binary-mixtures, J. Am. Chem. Soc., 1989, 111(22), 8533-8534.

12 T. Kato and J. M. J. Frechet, Stabilization of a liquidcrystalline phase through noncovalent interaction with a polymer side-chain, Macromolecules, 1989, 22(9), 38183819.

13 T. Kato, J. M. J. Frechet, P. G. Wilson, T. Saito, T. Uryu, A. Fujishima, C. Jin and F. Kaneuchi, Hydrogen-bonded liquid-crystals - novel mesogens incorporating nonmesogenic bipyridyl compounds through complexation between h-bond donor and acceptor moieties, Chem. Mater., 1993, 5(8), 1094-1100.

14 C. T. Imrie and P. A. Henderson, Liquid crystal dimers and higher oligomers: Between monomers and polymers, Chem. Soc. Rev., 2007, 36(12), 2096-2124.

15 C. T. Imrie, P. A. Henderson and G.-Y. Yeap, Liquid crystal oligomers: going beyond dimers, Liq. Cryst., 2009, 36(6-7), 755-777.

16 G. R. Luckhurst, Liquid-crystal dimers and oligomers experiment and theory, Macromol. Symp., 1995, 96, 1-26.

17 C. T. Imrie, F. E. Karasz and G. S. Attard, Comparison of the mesogenic properties of monomeric, dimeric, and sidechain polymeric liquid-crystals, Macromolecules, 1993, 26(3), 545-550.

18 G.-Y. Yeap, T.-C. Hng, S.-Y. Yeap, E. Gorecka, M. M. Ito, K. Ueno, M. Okamoto, W. A. K. Mahmood and C. T. Imrie, Why do non-symmetric dimers intercalate? The synthesis and characterisation of the -(4-benzylidene-substitutedaniline-4'-oxy)-(2-methylbutyl-4'-(4'-phen yl)benzoateoxy) alkanes, Liq. Cryst., 2009, 36(12), 1431-1441. 
19 C. T. Imrie, Non-symmetric liquid crystal dimers: How to make molecules intercalate, Liq. Cryst., 2006, 33(11-12), 1449-1454.

20 G. S. Attard, R. W. Date, C. T. Imrie, G. R. Luckhurst, S. J. Roskilly, J. M. Seddon and L. Taylor, Nonsymmetrical dimeric liquid-crystals - the preparation and properties of the $\alpha$-(4-cyanobiphenyl-4'-yloxy)- $\omega$-(4-n-alkylanilinebenzylidene-4'-o xy)alkanes, Liq. Cryst., 1994, 16(4), 529-581.

21 J. L. Hogan, C. T. Imrie and G. R. Luckhurst, Asymmetric dimeric liquid-crystals - the preparation and properties of the $\alpha$-(4-cyanobiphenyl-4'-oxy)- $\omega$-(4-n-alkylanilinebenzylidene-4'-oxy)hexanes, Liq. Cryst., 1988, 3(5), 645-650.

22 M. Cestari, S. Diez-Berart, D. A. Dunmur, A. Ferrarini, M. R. de la Fuente, D. J. B. Jackson, D. O. Lopez, G. R. Luckhurst, M. A. Perez-Jubindo, R. M. Richardson, J. Salud, B. A. Timimi and H. Zimmermann, Phase behavior and properties of the liquid-crystal dimer $1^{\prime}, 7^{\prime}$ bis(4-cyanobiphenyl-4'-yl) heptane: A twist-bend nematic liquid crystal, Phys. Rev. E: Stat., Nonlinear, Soft Matter Phys., 2011, 84(3), 031704.

23 P. A. Henderson and C. T. Imrie, Methylene-linked liquid crystal dimers and the twist-bend nematic phase, Liq. Cryst., 2011, 38(11-12), 1407-1414.

24 V. Borshch, Y. K. Kim, J. Xiang, M. Gao, A. Jakli, V. P. Panov, J. K. Vij, C. T. Imrie, M. G. Tamba, G. H. Mehl and O. D. Lavrentovich, Nematic twist-bend phase with nanoscale modulation of molecular orientation, Nat. Commun., 2013, 4, 2635.

25 C. T. Imrie, P. A. Henderson and J. M. Seddon, Nonsymmetric liquid crystal trimers. The first example of a triply-intercalated alternating smectic C phase, J. Mater. Chem., 2004, 14(16), 2486-2488.

26 P. A. Henderson and C. T. Imrie, Non-symmetric liquid crystal trimers, Liq. Cryst., 2005, 32(6), 673-682.

27 C. T. Imrie, D. Stewart, C. Remy, D. W. Christie, I. W. Hamley and R. Harding, Liquid crystal tetramers, J. Mater. Chem., 1999, 9(10), 2321-2325.

28 P. A. Henderson and C. T. Imrie, Liquid crystal tetramers: influence of molecular shape on liquid crystal behaviour, Liq. Cryst., 2005, 32(11-12), 1531-1541.

29 R. J. Mandle and J. W. Goodby, A Liquid Crystalline Oligomer Exhibiting Nematic and Twist-Bend Nematic Mesophases, ChemPhysChem, 2016, 17(7), 967-970.

30 R. J. Mandle and J. W. Goodby, Progression from nano to macro science in soft matter systems: dimers to trimers and oligomers in twist-bend liquid crystals, RSC Adv., 2016, 6(41), 34885-34893.

31 Y. Wang, G. Singh, D. M. Agra-Kooijman, M. Gao, H. K. Bisoyi, C. M. Xue, M. R. Fisch, S. Kumar and Q. Li, Room temperature heliconical twist-bend nematic liquid crystal, CrystEngComm, 2015, 17(14), 2778-2782.

32 T. Kato, A. Fujishima and J. M. J. Frechet, Self-assembly of a twin liquid-crystalline complex through intermolecular hydrogen bondings, Chem. Lett., 1990, (6), 919-922.

33 L. J. Yu and J. S. Pan, Mesophases induced by hydrogenbonds between non-mesogens - a di-proton acceptor and proton donors, Liq. Cryst., 1993, 14(3), 829-835.
34 M. J. Wallage and C. T. Imrie, Supramolecular dimeric liquid crystals. The liquid crystalline behaviour of mixtures of $\alpha$-(4pyridyloxy)- $\omega$ 4-(4-butylphenylazo)phenoxy alkanes and 4octyloxybenzoic acid, J. Mater. Chem., 1997, 7(7), 1163-1167.

35 N. P. S. Prabu and M. L. N. M. Mohan, Thermal Analysis, Calorimetric and Electrical Polarization Studies in Smectic $X^{*}$ Phase of Hydrogen-Bonded Ferroelectric Liquid Crystals, Mol. Cryst. Liq. Cryst., 2015, 606(1), 12-35.

36 C. V. Yelamaggad, G. Shanker, U. S. Hiremath and S. K. Prasad, Cholesterol-based nonsymmetric liquid crystal dimers: an overview, J. Mater. Chem., 2008, 18(25), 2927-2949.

37 S. M. Jansze, A. Martinez-Felipe, J. M. D. Storey, A. T. M. Marcelis and C. T. Imrie, A Twist-Bend Nematic Phase Driven by Hydrogen Bonding, Angew. Chem., Int. Ed., 2015, 54(2), 643-646.

38 H. Xu, N. Kang, P. Xie and R. B. Zhang, A new insight into the hydrogen-bonded liquid crystals built from carboxylic acids and pyridyl moieties, Mol. Cryst. Liq. Cryst., 2002, 373, 119126.

39 D. A. Paterson, A. Martinez-Felipe, S. M. Jansze, A. T. M. Marcelis, J. M. D. Storey and C. T. Imrie, New insights into the liquid crystal behaviour of hydrogenbonded mixtures provided by temperature-dependent FTIR spectroscopy, Liq. Cryst., 2015, 42(5-6), 928-939.

40 A. Martinez-Felipe and C. T. Imrie, The role of hydrogen bonding in the phase behaviour of supramolecular liquid crystal dimers, J. Mol. Struct., 2015, 1100, 429-437.

41 M. J. Plater, M. R. S. Foreman, T. Gelbrich and M. B. Hursthouse, Synthesis and characterisation of infinite di- and tri-nuclear zinc co-ordination networks with flexible dipyridyl ligands, J. Chem. Soc., Dalton Trans., 2000, (13), 1995-2000.

42 M. J. Frisch, et al., Gaussian 09 (Revision B.01), Gaussian Inc., Wallingford CT, 2010.

43 G. A. Jeffrey, An introduction to hydrogen bonding, Oxford University Press, New York and Oxford, 1997, p. 303.

44 M. Tarini, P. Cignoni and C. Montani, Ambient Occlusion and Edge Cueing for Enhancing Real Time Molecular Visualization, IEEE Trans. Visual. Comput. Graph., 2006, 12(5), 1237-1244.

45 R. Dennington, T. Keith and J. Millam, Gauss View, Version 5 , Semichem Inc, Shawnee Mission, KS, 2009.

46 C. T. Imrie, Laterally substituted dimeric liquid-crystals, Liq. Cryst., 1989, 6(4), 391-396.

47 P. A. Henderson, J. M. Seddon and C. T. Imrie, Methyleneand ether-linked liquid crystal dimers II. Effects of mesogenic linking unit and terminal chain length, Liq. Cryst., 2005, 32(11-12), 1499-1513.

48 D. Demus, H. Demus and Z. Zaschke, Flüssige Kristalle in Tabellen, VEB Deutscher Verlag für Grundstoff Industrie, Leipzig, 1974.

49 A. M. Shatalova, H. Kresse, G. A. Shandryuk, G. N. Bondarenko, S. A. Kuptsov and R. V. Talroze, The role of the alien proton acceptor on the formation of LC structure in $\mathrm{H}$-bonded monomeric and polymeric 
derivatives of alkoxybenzoic acids, J. Mol. Struct., 2004, 708(1-3), 7-14.

50 R. W. Date, C. T. Imrie, G. R. Luckhurst and J. M. Seddon, Smectogenic dimeric liquid-crystals - the preparation and properties of the alpha,omega-bis(4-normal-alkylanilinebenzylidine-4'-oxy)alkanes, Liq. Cryst., 1992, 12(2), 203-238.

51 J. Y. Lee, P. C. Painter and M. M. Coleman, Hydrogenbonding in polymer blends 4 . Blends involving polymers containing methacrylic-acid and vinylpyridine groups, Macromolecules, 1988, 21(4), 954-960.

52 A. Martinez-Felipe, A. G. Cook, M. J. Wallage and C. T. Imrie, Hydrogen bonding and liquid crystallinity of low molar mass and polymeric mesogens containing benzoic acids: a variable temperature Fourier transform infrared spectroscopic study, Phase Transitions, 2014, 87(12), 11911210.

53 S. E. Odinokov and A. V. Iogansen, Torsional gamma-(OH) vibrations, fermi resonance 2gamma- $(\mathrm{OH})$ - NU-isotopic effects in IR-spectra of H-complexes of carboxylic-acids with strong bases, Spectrochim. Acta, Part A, 1972, 28(12), 2343-2350.

54 S. L. Johnson and K. A. Rumon, Infrared spectra of solid 1-1 pyridine-benzoic acid complexes nature of hydrogen bond as a function of acid-base levels in complex, J. Phys. Chem., 1965, 69(1), 74-86.

55 A. G. Cook, A. Martinez-Felipe, N. J. Brooks, J. M. Seddon and C. T. Imrie, New insights into the transitional behaviour of methyl-6-O-(n-dodecanoyl)- $\alpha$-D-glucopyranoside using variable temperature FTIR spectroscopy and X-ray diffraction, Liq. Cryst., 2013, 40(12), 1817-1827.

56 A. G. Cook, J. L. Wardell, N. J. Brooks, J. M. Seddon, A. Martinez-Felipe and C. T. Imrie, Non-symmetric liquid crystal dimer containing a carbohydrate-based moiety, Carbohydr. Res., 2012, 360, 78-83.

57 A. M. Coats, D. W. L. Hukins, C. T. Imrie and R. M. Aspden, Polarization artefacts of an FTIR microscope and the consequences for intensity measurements on anisotropic materials, J. Microsc., 2003, 211, 63-66.

58 A. Martinez-Felipe, C. T. Imrie and A. Ribes-Greus, Study of Structure Formation in Side-Chain Liquid Crystal Copolymers by Variable Temperature Fourier Transform Infrared Spectroscopy, Ind. Eng. Chem. Res., 2013, 52(26), 8714-8721.
59 S. E. Odinokov, A. A. Mashkovsky, V. P. Glazunov, A. V. Iogansen and B. V. Rassadin, Spectral manifestations of intermolecular and interionic hydrogen-bonding in adducts of various acids with pyridine, Spectrochim. Acta, Part A, 1976, 32(6), 1355-1363.

60 M. S. Rosenber, A. a. Mashkovs, S. E. Odinokov and A. V. Iogansen, Torsional and librational frequencies of a$\mathrm{H}$ groups as related to $\mathrm{H}$-bonding energies, Spectrosc. Lett., 1972, 5(3-4), 75-80.

61 T. Kato, T. Uryu, F. Kaneuchi, C. Jin and J. M. J. Frechet, Hydrogen-bonded liquid-crystals built from hydrogenbonding donors and acceptors - infrared study on the stability of the hydrogen-bond between carboxylic-acid and pyridyl moieties, Liq. Cryst., 1993, 14(5), 1311-1317.

62 M. Petrov, E. Anachkova, N. Kirov, H. Ratajczak and J. Baran, IR spectroscopic investigations of peculiar behavior of $4, n-$ alkoxybenzoic acids in their mesomorphic state 1 . Nonaligned samples, J. Mol. Liq., 1994, 61(1-3), 221-230.

63 G.-Y. Yeap, F. Osman and C. T. Imrie, Non-symmetric dimers: effects of varying the mesogenic linking unit and terminal substituent, Liq. Cryst., 2015, 42(4), 543-554.

64 Z. Lu, P. A. Henderson, B. J. A. Paterson and C. T. Imrie, Liquid crystal dimers and the twist-bend nematic phase. The preparation and characterisation of the $\alpha, \omega$-bis(4cyanobiphenyl-4'-yl) alkanedioates, Liq. Cryst., 2014, 41(3), 471-483.

65 T.-N. Chan, Z. Lu, W.-S. Yam, G.-Y. Yeap and C. T. Imrie, Non-symmetric liquid crystal dimers containing an isoflavone moiety, Liq. Cryst., 2012, 39(3), 393-402.

66 H.-C. Lee, Z. Lu, P. A. Henderson, M. F. Achard, W. A. K. Mahmood, G.-Y. Yeap and C. T. Imrie, Cholesteryl-based liquid crystal dimers containing a sulfursulfur link in the flexible spacer, Liq. Cryst., 2012, 39(2), 259-268.

67 P. Painter, C. Cleveland and M. Coleman, An infrared spectroscopic study of p-n-alkoxybenzoic acids, Mol. Cryst. Liq. Cryst., 2000, 348, 269-293.

68 E. I. Efremova, A. A. Shiryaev, Z. A. Kydryashova, L. A. Nosikova, S. A. Syrbue and V. V. Chernyshev, Study of phase behavior in a system of linear hydrogen-bonded carboxylic acid homologues, Phase Transitions, 2015, 88(5), 503-512. 